\title{
Abstracts of the Third International Congress on Treatment of Dystonia, 4-7 May 2016, Hannover, Germany
}

(C) The Author(s) 2016. This article is published with open access at Springerlink.com

\section{Foreword from the Organisers}

Dystonias are amongst the most common movement disorders. Botulinum toxin and deep brain stimulation have revolutionized their therapy. There are, however, still considerable unmet therapeutic needs.

For this reason a series of conferences have been initiated on this topic. Two congresses have already taken place, in 2010 and 2013. Both received an extremely warm welcome from the medical and scientific community. We were therefore very enthusiastic about organising a third congress, from May 4th to 7th, 2016. Pre-meeting satellite events included the Second International Conference on Rehabilitation of Dystonia and the newly introduced First International Conference on Dystonia in Sports.
The intense three-and-a-half day program consisting of state-ofthe-art lectures, special lectures, workshops, industry symposia and a busy industry exhibition this time lured more than 500 participants from over 40 countries and all continents to Hannover. With a faculty of more than 80 world-leading experts from neurology, neurosurgery, neuropediatrics, neurogenetics, allied health professions and basic sciences this was, once again, the leading congress on dystonia.

The following abstracts accompanied the posters presented at the Congress. They cover a wide spectrum of interesting topics and give a comprehensive overview of the latest research in dystonia.

Enjoy reading and stay with us to further develop this open platform for all who take care of people with dystonia.

Eckart Altenmüller Dirk Dressler Joachim K Krauss 


\section{Clinical effects for cervical dystonia using a device inducing hanger reflex - multicenter trial}

Asahi T. ${ }^{1}$, Sato M. ${ }^{2}$, Nakamura T. ${ }^{2}$, Kon Y. ${ }^{2}$, Kajimoto H. ${ }^{2}$, Oyama G. ${ }^{3}$, Hayashi A. ${ }^{4}$, Tanaka K. ${ }^{5}$, Nakane T. ${ }^{6}$, Takeshima T. ${ }^{7}$, Fujii M.

${ }^{1}$ Kanazawa Neurosurgical Hospital, Department of Neurosurgery, Ishikawa, Japan, ${ }^{2}$ University of Electro-Communications, Department of Informatics, Tokyo, Japan, ${ }^{3}$ Juntendo University, Department of Neurology, Tokyo, Japan, ${ }^{4}$ Juntendo University Urayasu Hospital, Department of Rehabilitation, Chiba, Japan, ${ }^{5}$ Saiseikai Matsuyama Hospital, Department of Neurosurgery, Ehime, Japan, ${ }^{6}$ Nagasaki Kawatana Medical Center, Department of Neurology, Nagasaki, Japan, ${ }^{7}$ Tominaga Hospital, Department of Neurology, Osaka, Japan, ${ }^{8}$ Yamaguchi University, Department of Neurosurgery, Yamaguchi, Japan

Background: When the head is encircled with a wire clothes hanger and the unilateral fronto-temporal region is compressed, the head rotates unexpectedly. As the mechanism is unclear, however, we have temporarily named this phenomenon as the hanger reflex (HF). We previously reported that a HR occurs in more than $90 \%$ of normal subject, and experience a case wherein this phenomenon was applied to treat cervical dystonia (CD).

Objectives: Investigate the clinical effects for $\mathrm{CD}$ using a device induce HR by a multicenter clinical trial.

Methods: This study included totally 19 patients (12 men and 7 women) aged 23-86 years with rotational type of CD. An elliptical device induce the HR was applied to each subject's head. Patients applied the device more than $30 \mathrm{~min} /$ day for 3 months. Severity score (part 1) of Toronto western torticollis scale (TWSTRS) with and without the device were evaluated before and after 3 months trial.

Results: Mean TWSTRS part I scores without the device before and after the trial were 16.6 and 14.7, and the scores with the device were 15.0 and 13.6, respectively. The scores without the device were significantly decreased compared to before and after the trial $(\mathrm{P}=0.020)$, and the scores after attaching the device at the starting the trial were significantly decreased $(\mathrm{P}=0.001)$.

Conclusions: This preliminary clinical trial suggested that abnormal head rotation of the $\mathrm{CD}$ was restricted by the device even without attaching the device after the trial. This device has potential to be a novel, less invasive, and inexpensive treatment for CD.

\section{Improvement of table tennis dystonia by stereotactic ventro-oral thalamotomy}

Asahi T. ${ }^{1}$, Taira T. ${ }^{2}$, Ikeda K. ${ }^{1}$, Yamamoto J. ${ }^{1}$, Sato S. ${ }^{1}$

${ }^{1}$ Kanazawa Neurosurgical Hospital, Department of Neurosurgery, Ishikawa, Japan, ${ }^{2}$ Tokyo Women's Medical University, Department of Neurosurgery, Tokyo, Japan

Background: Focal task-specific dystonia including writer's cramp and musician's cramp is a kind of dystonia. Stereotactic ventro-oral (Vo) thalamotomy has been performed for these movement disorders with excellent outcome. However, there is no report on treatment for already known sport-related dystonia by stereotactic surgery in the literature. We treated a patient with table tennis dystonia by $\mathrm{Vo}_{0}$ thalamotomy and evaluated the outcome.

Case report: A 20-year-old female left-handed table tennis player has experienced difficulty of hitting a ping-pong ball. She has been practicing over $4 \mathrm{~h}$ every day for 12 years, and could participate in national tournaments. She experienced abnormal flexion of the left wrist when hitting a ball at 19 years of age. The abnormal movement appeared on the forehand stroke and, then, on the backhand, and finally she could not continue playing. She was diagnosed as focal task-specific dystonia by neurologists and was not recovered with medication. She visited our hospital and underwent right Vo thalamotomy. The surgery was performed under local anesthesia with the patient swinging a racket during stimulation and coagulation of the thalamus. Her symptoms improved intraoperatively just after Vo thalamotomy. She was completely recovered from movement disorder, and allowed to participate in tournament again.

Conclusion: To our knowledge, this is the first report of a case demonstrating the effectiveness of Vo thalamotomy not only for writer's cramp and musician's cramp, but also for sport-related dystonia.

\section{Treatment of muscle dystonia with CT-guided injections of botulinum toxin type A: technique, indications and perspectives}

$\underline{\text { Balbert A. }}{ }^{1,2}$, Myakotnykh V. ${ }^{1,2}$

${ }^{1}$ Ural State Medical University, Gerontology, Ekaterinburg, Russian Federation, ${ }^{2}$ Sverdlovsk Regional Hospital for Veterans of War, Neurology, Ekaterinburg, Russian Federation

Clinical experience: The use of botulinum toxin type A for the treatment of patients with different types of muscle dystonia has shown the treatment to be not always effective. The most difficult injections are into deep muscles. Ultrasound cannot differentiate muscles lying deeper than $5 \mathrm{~cm}$ while blind needle insertion can damage blood vessels, nerves and internal organs. Experience has shown the best method to control the accuracy of botulinum toxin injection is with Computed Tomography (CT). Our extensive experience in making CT-guided injections into: $\mathrm{m}$. oblique inferior; $\mathrm{m}$. psoas major and $\mathrm{m}$. piriformis has proven successful in minimizing patient pain while optimizing doctors' time and increasing patient satisfaction.

The advantages of this method are its high accuracy and absolute certainty in making injects into target muscles. This significantly increases the effectiveness of treatment for patients with deep muscle hyperactivity. The accuracy of making injections is a skill I teach.

We suggested diagnostic and treatment technique for CT guided BTA injections in certain types of dystonia. Successful implementation improved treatment outcomes by about $30 \%$ and reduced amounts of botulinum toxin needed by about $25 \%$ based upon comparisons made in our own hospital over 2 years. The results were achieved due to proper selection of the target muscle and precise needle placement, which I teach.

Currently, our technique has been applied in the Veterans Hospital (Ekaterinburg, Russia). Study is ongoing to expand the applications of CT guided BTA injections for new indications.

In the past year over 20 workshops on various techniques of injections have been given in hospitals all over Central Russia.

\section{Exploring the role of healthcare in the construction of lay experts' experience of physical disability: a qualitative interview study of dystonia patients in England}

Bernstein C.J. ${ }^{1}$, Boardman F. ${ }^{1}$, Griffiths F. ${ }^{1}$, Sandhu H. ${ }^{2}$ 
${ }^{1}$ University of Warwick, Division of Health Sciences, Warwick Medical School, Coventry, United Kingdom, ${ }^{2}$ University of Warwick, Clinical Trials Unit, Warwick Medical School, Coventry, United Kingdom

Health scientists have frequently emphasised the importance of exploring patients' experiences of healthcare for improving services and quality of life (QoL). Such experiences, including individuals' perceptions of healthcare provision, have been explored in people with dystonia using mainly quantitative measures. Yet, standardised QoL measures have been criticised for neglecting to capture the nuanced ways in which patients living with dystonia draw on their illness experiences to make sense of complex healthcare decisions. Consequently, the aim of this study is to explore how patients utilise their embodied (physical and socio-emotional) experiences to promote, resist, and challenge healthcare practices.

This study reports on group and individual interview data with 42 adults with multiple types of dystonia. Twenty-seven people attended local dystonia support groups and two were patients. In addition, secondary analysis was conducted on twenty-one interviews with thirteen patients, originally recruited for a feasibility study exploring the self-management of dystonia. The data were thematically analysed for recurring patterns and relationships as well as deviant cases.

The interviews revealed the significance of participants' embodied knowledge for negotiating emotional healthcare experiences and decisions. Many participants reported difficulties in discussing their experiences with health professionals, utilising their embodied knowledge to seek out other forms of health-related information, namely from support groups and the internet. Nonetheless, participants drew productively on medical information when it aligned with their own experiences and understandings of dystonia.

Using the findings of this study, I will argue that dystonia is experienced holistically by those who live with it and subsequently, I will move beyond a clinical reading of their diagnostic type to incorporate the lived reality of dystonia. The implications of these findings suggest that health professionals should recognise the importance of embodied experiences to patients' healthcare decisions and experiences as well as the role of technology in shaping their accounts.

\section{0 years retraining for pianists with focal dystonia: retrospective and perspectival methodological analysis}

\section{Boullet L. ${ }^{1}$ \\ ${ }^{1}$ Hannover Institute of Music Physiology \& Musician's Medicine (external member), Hannover, Germany}

Introduction: over the past 20 years, the retraining of pianists with focal dystonia has become a reliable method to significantly improve their condition. Due to a close collaboration with the Hannover Institute of Music Physiology \& Music Medicine, the integration of fundamental research and the combination of additional medications such as anticholinergic substances or Botox, one has achieved for the majority of patients substantial relieves up to a professional playing level. However, we still face crucial issues such as the general duration of the retraining (generally 2 years or more), or the obligation to partly change highly automatized motor programs. In order to still improve retraining approaches as well as to potentially give new impulses to medical research, it is important to statistically evaluate the method and find out what are the common retraining schemes used among patients.
Method: using data protocols of more than 100 pianists retrained, it is possible to establish basic statistics that enable us to draw a more precise picture of the retraining. Starting with the prevalence of compensation patterns and combining it with corresponding exercises strategies that proved being successful, it becomes possible to establish new schemes of focal hand dystonia phenomenon among musicians. In addition to this method, experience has shown that a vast variety of sensory-motor tricks still remain a valuable strategy to regain control and reprogram the hand. Their systematic listing matched with the correlative compensation patterns completes this new draft after which the retraining method is now designed. It suggests that lack of sensory-motor feedback plays a major role in the emergence of compensation patterns together with so called predictive mechanisms of movements.

Conclusion: it has become a central issue for patients to better understand the mechanisms of focal hand dystonia. The newly established scheme gives them a clearer picture of how FD may affect their complex movement patterns. It is an obvious help in their retraining strategies, not only in the deprogramming phase (reducing compensations and lowering general muscle tone), but also in the following reprogramming phase (acquiring new motor patterns for basic technical approach such as scale playing) and up to repertoire playing.

\section{Deep brain stimulation for dystonia in the context of medtronic-supported, investigator-sponsored research}

\author{
Brionne T.C. ${ }^{1}$, Appenrodt P. ${ }^{1}$, Mullett K. ${ }^{1}$, Sarem-Aslani A. ${ }^{1}$ \\ ${ }^{1}$ Medtronic, Clinical Research \& Innovation, Tolochenaz, \\ Switzerland
}

Introduction: To foster the generation of clinical evidence, the Medtronic Neuromodulation Clinical Research (CR) organization has been supporting European investigator-sponsored research programs (External Research Programs; ERPs) since the late 1990s. The scientific output of these ERPs included doctoral theses and congress presentations, but peer-reviewed publications remain the gold standard of evidence-based medicine. The purpose of this work is to quantify this publication output based on Medtronic support, and to characterize the Deep Brain Stimulation (DBS) for Dystonia literature compared to the overall Neuromodulation therapies and to other DBS research areas.

Methods: Publications were spontaneously submitted by investigators to the CR team, and to ensure thoroughness, PubMed and Embase searches were performed using principal investigator names. Full publications were downloaded and individually checked for relevance to each ERP. Publication dates ranged from 1999 to 2016. Journal impact factors retained were the latest available values, either through direct disclosure by the journal or online databases.

Results and discussion: Medtronic-supported Neuromodulation ERPs generated over 300 peer-reviewed publications. DBS for Movement Disorders accounted for over $25 \%$ of this total. DBS for Dystonia accounted for around $10 \%$ of this subgroup, and included several publications from clinical trials with randomized controlled assessments published in high impact journals (New England Journal of Medicine, Lancet Neurology). Overall, studies focused on short and long term clinical outcomes of DBS for generalized, segmental and cervical dystonia. Few studies included disease-specific quality of life scales or mode of action research. These publications are a key indicator of the scientific productivity of Medtronic's partnership with European investigators in the area of DBS. 


\section{Multimodal surgical treatment of generalized dystonia}

Brodacki B. ${ }^{1}$, Kmiec T. ${ }^{2}$, Koziara H. ${ }^{3,4}$, Bonicki W. ${ }^{1,3}$, Mandat T. ${ }^{3,4}$

${ }^{1}$ Military Medical Institute, Neurology, Warszawa, Poland, ${ }^{2}$ Institute Centrum Zdrowia Dziecka, Neurology, Warszawa, Poland, ${ }^{3}$ Maria Sklodowska-Curie Oncology Memorial Center, Neurosurgery, Warszawa, Poland, ${ }^{4}$ Institute of Psychiatry and Neurology, Neurosurgery, Warszawa, Poland

Objective: Evaluation of effectiveness and safety of multimodal stereotactic procedures (lesioning and deep brain stimulation (DBS)) and multifocal (globus pallidus pars interna (GPi) and subthalamic nucleus (STN)) treatment of generalized dystonia (GD).

Background: Thalamotomy and pallidotomy are the primary stereotactic methods of GD treatment. DBS is a surgical method of choice for various movement disorders treatment that include GD. Multimodal and multifocal DBS was involved in the movement disorders treatment to achieve better results, but no analysis has been conducted yet.

Methods: Seven GD patients with history of bilateral GPi DBS or thalamotomy or pallidotomy were qualified for further surgical treatment. All of them demonstrated significant improvement that vanished few years after surgery. Five of them, who underwent lesioning in the past were qualified for DBS: GPi (3 patients) and STN (2 patient). Two patients, who underwent GPi stimulation in past were qualified for multifocal stimulation- additional STN DBS.

Results: Multimodal and multifocal DBS have caused sustained improvement in dystonic movement (measured with GDS) that lasted in two to 5 years follow-up. No surgical or stimulation related complications were reported.

Conclusions: Multimodal: lesioning and DBS and combined, multifocal GPi and STN DBS might and should be considered when dystonic symptoms aggravate over time.

\section{Long lasting cerebral functional connectivity (FC) changes of a rehabilitation perceptive integrated approach (PIA) associated with botulinum toxin $A$ in cervical dystonia}

\author{
Castagna A. ${ }^{1}$, Sciumè L. ${ }^{1}$, Costantini I. ${ }^{1}$, Ramella M. ${ }^{1}$, Montesano \\ A. $^{1}$, Baglio F. ${ }^{1}$ \\ ${ }^{1}$ Don Gnocchi Foundation IRCCS Santa Maria Nascente, Milano, \\ Italy
}

Introduction: Cervical dystonia (CD) is characterized by involuntary, abnormal movements and postures of the head and neck. It is currently underlined that dystonia is a network disease between cerebellum, basal ganglia and cortex. Efficacy of Botulinum toxin (BoNTA) has been demonstrated and there are several data that physical therapy can improve treatment outcome acting on sensory motor re-learning.

Methods: Five CD patients were included in the study and underwent BoNTA injection and a rehabilitation PIA (eighteen 45 min sessions three times a week). They were evaluated before (T0) and after treatments (T1 at 6 week and $\mathrm{T} 2$ at 3 months) with Toronto Western Spasmodic Torticollis Rating Scale (TWSTRS) and resting state functional MRI. Imaging data of CD patients were compared with 19 age-matched healthy subjects.

Data were statistically analyzed with the repetitive measures ANOVA corrected with Bonferroni.
Results and discussion: Data showed statistically significant improvement in TWSTRS at T1 and persistence of the result at T2 $(\mathrm{P}<0.05)$. AT $\mathrm{T} 1$ patients showed $\mathrm{FC}$ values similar to normal subject FC pattern. At follow up FC between sensory motor network $(\mathrm{SMN})$ and visual (VIS), pre-cuneal (PCU) and default mode (DM) networks were still modified.

Interestingly changes between FC and clinical changes in TWSTRS (towards lower scores) had a statistically significant correlation.

In conclusion: BoNTA associated to PIA program is efficacious in restoring SM FC in CD patients at T1. Moreover the clinical prolonged effect can be due to the PIA that could facilitate the FC between sensory motor and frontal parietal executive areas. This could correlate with the restored motor control at $\mathrm{T} 2$ as demonstrated by TWSTRS scores. Nevertheless at T2 there is a return towards altered connectivity between sensory motor and visual cortex as shown in $\mathrm{T} 0$.

\section{A new perceptive integrated approach (P.I.A.) based on motor learning principles associated to Botulinum Toxin Treatment (BoNT-A) in Cervical Dystonia (CD)}

\author{
Castagna A. ${ }^{1}$, Crippa A. ${ }^{1}$, Giacobbi G. ${ }^{1}$, Corrini C. ${ }^{1}$, Sciumè L. ${ }^{1}$, \\ Ramella M. ${ }^{1}$, Montesano A. ${ }^{1}$ \\ ${ }^{1}$ Don Gnocchi Foundation IRCCS Santa Maria Nascente, Milano, \\ Italy
}

Introduction: $\mathrm{CD}$ is a movement disorder characterized by involuntary and abnormal movements and head and neck postures that affect daily and social life activities. The CD pathophysiologic bases are faulty sensory-motor integration and impaired motor planning. BoNT efficacy has been demonstrated and there are some indications that standard physical therapy can improve symptoms. Approaches applying sensory-motor re-learning has been suggested as an integration to standard treatment.

The PIA motor exercises have fixed temporal and spatial criteria (open/closed eyes, laying/sitting/standing) (SaM Method) that can be combined to obtain a tailored rehabilitation program.

The PIA also uses sensory (visual, acoustic and vibratory) biofeedback during the exercises The devices (inclinometer, goniometer, laser) help the patient to improve kinematic and proprioceptive consciousness and to re-learn correct head neck and trunk posture and movements.

Methods: Ten patients affected by CD were included in the study. All patients were evaluated before (T0) at 6 weeks (T1) and 3 months (T2) after the BoNT-A treatment. CD patients underwent eighteen 45 min PIA sessions three times a week for 6 weeks. The outcome measures was Toronto Western Spasmodic Torticollis Rating Scale (TWSTRS). Data were then analyzed with the Wilcoxon rank test. Results and discussion: Data showed significant improvement in TWSTRS at T1 $(\mathrm{P}<0.05)$ and maintain the result at T2 $(\mathrm{P}<0.05)$. The PIA has so far showed promising prolonged effects in the posture and motor skills in patients with $\mathrm{CD}$ by restoring motor control and daily life strategies. Further investigation is necessary, but the PIA appeared to be a useful complementary treatment for patients affected by $\mathrm{CD}$.

\section{Treatment satisfaction with botulinum toxin among patients with cervical dystonia in Spain}

Martí M.J. ${ }^{1}$, Catalán M.J. ${ }^{2}$ 
${ }^{1}$ Department of Neurology, Hospital Clínico Provincial, Barcelona, Spain, ${ }^{2}$ Department of Neurology, Hospital Clínico San Carlos, Madrid, Spain

Introduction: The recommended treatment interval between botulinum neurotoxin (BoNT) injections for focal dystonia is generally $\geq 12$ weeks. However, symptom recurrence before re-injection is common. This 2014 survey evaluated satisfaction with BoNT treatment regimens among patients with cervical dystonia (CD) in Spain. Methods: The survey included patients with $\mathrm{CD}$ who had received at least three BoNT treatments. Patients were screened for eligibility in telephone interviews and subsequently participated in an online survey 7-10 weeks after their last BoNT treatment. Participants rated their satisfaction with BoNT treatment on a 1-10 scale $(1=$ not at all satisfied; $10=$ fully satisfied); ratings were obtained for satisfaction at the time of interview, and as recalled at the peak of treatment effect and directly before re-injection.

Results and discussion: Of the 93 participants, $44 \%$ were female. The mean age was 51.2 years and the mean duration of $\mathrm{CD}$ was 10.1 years. Patients had received BoNT treatment for a mean 8.7 years with a mean treatment interval of 13.2 weeks. However, a majority of patients $(64 \%)$ reported a preference for treatment intervals $<12$ weeks, and $32 \%$ for treatment intervals $<10$ weeks. At the time of interview, $47 \%$ of patients were very satisfied (rating of 8-10) with BoNT treatment, compared with $78 \%$ at the peak of treatment effect and $17 \%$ directly before re-injection. The most frequent reasons patients recalled being given by their physician for the interval chosen were 'to avoid antibody formation' (70\%) and 'because shorter intervals are not recommended' (55\%).

In conclusion, the decline in satisfaction from high levels at peak efficacy to low levels before re-injections, and the high proportion of patients who would prefer $<12$-week treatment intervals $(64 \%)$ suggest that patient satisfaction with BoNT treatment for CD may be improved with more flexible, individualised injection intervals.

\section{Status dystonicus: Refining the condition and its management based on forty cases}

Cif L. ${ }^{1}$, Nerrant E. ${ }^{1}$, Roujeau T. ${ }^{1}$, Milesi C. ${ }^{2}$, Cambonie G. ${ }^{2}$, Ros M. ${ }^{1}$, Chan Seng E. ${ }^{1}$, Cyprien F. ${ }^{1}$, Sanrey E. ${ }^{1}$, Gonzalez Martinez V. ${ }^{1}$, Coubes P.

${ }^{1}$ Université de Montpellier; CHRU Montpellier, Neurochirurgie, Montpellier, France, ${ }^{2}$ Université de Montpellier; CHRU Montpellier, Pédiatrie Néonatale et Réanimation, Montpellier, France

Introduction: Despite better knowledge of various forms of dystonia and their pathogenesis, only sparse information is available for the understanding and prognosis of increasingly worsening dystonic conditions labelled status dystonicus (SD).

Methods: We studied a group of dystonia patients who exhibited, during disease course, at least one episode fulfilling the previously proposed criteria for SD. Our aim is to pinpoint indicators of progression towards SD and to further report on the long term evolution and therapeutics, with highlight on the intensive care unit management and deep brain stimulation effects.

Three dimensions were explored and the following parameters documented for each of them:

- Clinical characteristics (birth parameters, family history, etiology, age at disease onset, time frame of disease progression and of worsening towards SD, symptom type, distribution and severity, etiology, medication and triggering factors of SD);

- Morphological changes documented by MRI;
- Modality of decompensation at the time of SD (hyperthermia, rhabdomyolisis, dysphagia, weight loss, percutaneous gastrostomy, respiratory failures, renal failure, hepatic failure, pain, sleep alteration).

Results and discussion: Forty dystonia patients ( 21 female) followed for SD in our Center were included. Age at SD ranged from 5 to 45.5 years. Dystonia was generalized in all but one patient previous to the SD and etiology heterogeneous, remaining unknown despite extensive investigation in 14 subjects. Dystonia was related to mutations in TOR1A gene in 4 , to PANK2 gene in other 4 and to HPRT1 gene in 3 subjects respectively. The most frequently identified triggering factors were therapy discontinuation or new drug administration and, inadvertent DBS discontinuation and DBS system dysfunction during follow up for DBS. Twenty-six patients presented DS previous to DBS administration and 22 subjects under DBS. Eight patients died during the follow-up. The most efficient therapeutic management was DBS with the longest follow after SD of 19.5 years.

\section{Economics of botulinum toxin therapy: Influence of the abobotulinumtoxin A package size on the costs of botulinum toxin therapy}

\author{
$\underline{\text { Dressler D. }}{ }^{1}$, Adib Saberi F. ${ }^{1}$ \\ ${ }^{1}$ Hannover Medical School, Movement Disorders Section, \\ Department of Neurology, Hannover, Germany
}

AbobotulinumtoxinA (Dysport ${ }^{\circledR}$ ) was distributed for many years in vials containing 500MU (D500). Recently a new 300MU vial (D300) was additionally introduced (introduction). We wanted to explore whether more differentiated package sizes allow for more economic use of Dysport ${ }^{\circledR}$ in a large neurological botulinum toxin (BT) outpatient clinic ( $>9000$ standard vials/a).

The study followed a retrospective chart review design based on our digital BT therapy data bank. All patients receiving Dysport ${ }^{\circledR}$ exclusively in a constant dose during the observation period (introduction \pm 7 months) were included. Economic calculations are based on Dysport ${ }^{\circledR}$ prices as officially advertised in Germany. Sharing of vials between patients was not allowed.

Altogether 83 patients (51 with dystonia, 25 with spasticity, 3 with hemifacial spasm, 4 with other diagnoses) were included in this study. The total amount of MU used before and after introduction was 102525 , the amount prescribed 138000MU and 116300MU ($21700 \mathrm{MU},-15.7 \%$ ), the costs $€ 146103$ and $€ 125250$ ( $-€ 20853$, $-14.3 \%$ ). The price of D500 before and after introduction was $€ 529.36$, for D300 $€ 339.71$. The D500 price for $1 \mathrm{MU}$ before and after introduction is $€ 1.0587$, the $\mathrm{D} 300$ price for $1 \mathrm{MU} € 1.1324$ ( $€ € 0.073$, $+7.0 \%$ against D500).

More flexible packaging reduces drug costs for BT therapy considerably. Introduction of smaller packaging sizes is technically possible and should be encouraged. Extra costs for registration and logistics are moderate. Further cost reductions may be possible by introduction of even smaller packaging sizes. They can be calculated based on our model.

\section{Mouse diaphragm assay confirms identical potency labelling for onabotulinumtoxinA (Botox ${ }^{\circledR}$ ) and incobotulinumtoxin $A\left(\operatorname{Xeomin}^{\circledR}\right)$}

$\underline{\text { Dressler D. }}{ }^{1,2}$, Bigalke H. ${ }^{1,2}$ 
${ }^{1}$ Hannover Medical School, Movement Disorders Section, Department of Neurology, Hannover, Germany, ${ }^{2}$ Toxogen GmbH, Hannover, Germany

Labelling of botulinum toxin drugs is an ongoing matter of debate. We wanted to test the potency labelling of onabotulinumtoxinA $\left(\right.$ Botox $\left.{ }^{\circledR}\right)$ and incobotulinumtoxinA $\left(\mathrm{Xeomin}^{\circledR}\right)$ in the mouse diaphragm assay (MDA).

For this, 5 vials of unexpired onabotulinumtoxinA 100MU (Botox ${ }^{\circledR}$, Pharm-Allergan, Frankfurt/M, Germany, 53396EW15C/ C3497C3/expiry date 10/2016, 53396W15C/C3510C3/expiry date 11/2016) and 3 vials of unexpired incobotulinumtoxinA 100MU (Xeomin $^{\circledR}$, Merz Pharmaceuticals, Frankfurt/M, Germany, 370163/expiry date 12/2016, 370163/expiry date 12/2016) were reconstituted with $2.0 \mathrm{ml} 0.9 \% \mathrm{NaCl} / \mathrm{H}_{2} \mathrm{O}$ without any other additives using normal clinical equipment. $1.8 \mathrm{ml}$ of each sample was added to the organ chamber of a quality controlled MDA set-up and halfparalysis times were recorded.

Xeomin ${ }^{\circledR}$ samples produced half-paralysis times of 63,61 and $71 \mathrm{~min}$ (mean $\pm \mathrm{SD}, 66.0 \pm 7.0 \mathrm{~min}$ ). Half-paralysis times of Botox $^{\circledR}$ samples were $70,71,80,71$ and 68 min (mean $\pm S D$, $72.0 \pm 4.6 \mathrm{~min})$. There was no statistical significant difference between both groups ( $\mathrm{p}=0.133$, Welch's $t$ test).

This study shows identical potency labelling for Botox ${ }^{\circledR}$ and Xeomin ${ }^{\circledR}$. It confirms previous results obtained in a mouse lethality assay and in various clinical studies.

\section{Safety of botulinum toxin short interval therapy using incobotulinumtoxin A}

Dressler D. ${ }^{1}$, Adib Saberi F. ${ }^{1}$

${ }^{1}$ Hannover Medical School, Movement Disorders Section, Department of Neurology, Hannover, Germany

The therapeutic efficacy of botulinum toxin (BT) can be completely blocked by formation of BT antibodies (BTAB) thus producing antibody-induced therapy failure (ABTF). One of the risk factors for this is the interval between two subsequent injection series. To prevent BTAB formation it is universally recommended not to use interinjection intervals of less than 12 weeks. However, BT's therapeutic efficacy may be considerably shorter than this interval thus causing considerable reduction of quality of life. We wanted to study whether BT therapy with interinjection intervals of less than 12 weeks (short interval therapy, SIT) would be immunologically and otherwise safe. To minimise the risk of $\mathrm{BTAB}$ formation we used incobotulinumtoxin A which has a particularly low antigenicity.

Altogether 30 patients (age 59.2 \pm 13.5 years. 19 females, 11 males) with different dystonias were included in this study. They received SIT with incobotulinumtoxinA (Xeomin ${ }^{\circledR}$, Merz Pharmaceuticals, Frankfurt/M, Germany) at interinjection intervals of $69.0 \pm 8.1$ days (equal 9.9 weeks or 2.2 months, min. $48.9 \pm 2.4$ days) for $14.3 \pm 2.9$ injection series (equal $906 \pm 169$ days or $2.5 \pm 0.5$ years) in a dose of $259 \pm 159 \mathrm{MU}$ (max. $670 \pm 144.4 \mathrm{MU}$ ). None of the patients showed signs of ABTF, unusual BT effects or increased adverse effects.

Information provided by this study confirms safety of SIT. With a considerable percentage of patients hitherto undertreated for prolonged periods of time with BT therapy applying 12 weeks intervals, SIT may substantially improve the quality of life for those patients. Whether SIT is also safe with conventional BT drugs is something that still needs to be tested.

\section{Strategies to decrease injection site pain in botulinum toxin therapy}

\author{
Paracka L. ${ }^{1}$, Kollewe K. ${ }^{1}$, Wegner F. ${ }^{1}$, Dressler D. ${ }^{1}$ \\ ${ }^{1}$ Hannover Medical School, Movement Disorders Section, \\ Department of Neurology, Hannover, Germany
}

Botulinum toxin (BT) is now used for numerous indications including dystonias, spasticity, cerebral palsy, hyperhidrosis, cosmetics and chronic migraine. It has to be injected into its target tissues thus causing injection site pain (ISP). We wanted to compare the efficacy of various analgesic interventions suggested for ISP reduction.

In 13 healthy controls pain thresholds in the fingertips II and III bilaterally were determined in the Mechanical Pain Threshold Test (MPTT) and the Repetitive Pain Stimulation Test (RPST) at baseline and under nitrous oxide/oxygen (NOO), ice spray (IS), local anaesthetic cream (LAC) and forearm ischaemia (FI).

All analgesic interventions studied produce statistically significant and robust analgesic effects in the MPTT and the RPST. NOO had stronger analgesic effects than the other interventions, although this superiority was statistically significant only in the RPST and not against IS.

Additionally considering duration, localisation and penetration depth of the analgesic effect, hyperhidrosis treatment may benefit from NOO, IS and LAC. In palmar hyperhidrosis FI is possible and also reduces BT washout. Cosmetic indications may also benefit from NOO and LAC. For BT therapy of spasticity, dystonia and tremor only NOO may offer intramuscular analgesic effect. Its systemic and prolonged effect is also an advantage in wide-spread injections in several body parts.

Future studies are necessary to test the influence of penetration depth and combinations of analgesic interventions.

\section{Botulinum toxin therapy for hyperhidrosis: reduction of injection site pain by nitrous oxide/oxygen mixtures}

\author{
Paracka L. ${ }^{1}$, Kollewe K. ${ }^{1}$, Dressler D. ${ }^{1}$ \\ ${ }^{1}$ Hannover Medical School, Movement Disorders Section, \\ Department of Neurology, Hannover, Germany
}

Injection site pain (ISP) reduces compliance of botulinum toxin (BT) therapy considerably. We wanted to study whether nitrous oxide/oxygen (NOO, Livopan ${ }^{\circledR}$, Linde Gas Therapeutics, Unterschleißheim, Germany) can reduce ISP in patients receiving intracutaneous BT injections for axillary of palmar hyperhidrosis (HH).

The study followed an open-label design comparing intraindividually ISP in both axillae and/or both palms when NOO was applied or not during BT injections. BT efficacy was measured by the Hyperhidrosis Disease Severity Scale (HDSS) and by a 4-point SelfAssessment Scale (SAS). ISP was documented by a Visual Analogue Scale (VAS) and the Verbal Scale of Pain Intensity (VSPI), adverse effects by a Structuralised Interview (SI).

Altogether 13 patients (age $34.1 \pm 12.4$ years, 9 females, 4 males) were studied. $11 \mathrm{BT}$ treatments were for biaxillary and 3 for bipalmar $\mathrm{HH}$. BT reduced biaxillary $\mathrm{HH}$ from HDSS $3.7 \pm 0.5$ to $1.0 \pm 0$ and bipalmar $\mathrm{HH}$ from $3.6 \pm 0.6$ to $1.0 \pm 0$. All patients reported ISP reduction by NOO. In axillary $\mathrm{HH}$ NOO reduced ISP from $55.7 \pm 12.7$ to $12.8 \pm 7.5$ on the VAS $(\mathrm{p}<0.05)$ and from $4.1 \pm 0.3$ to $0.7 \pm 0.5$ on the VSPI $(\mathrm{p}<0.05)$, in bipalmar $\mathrm{HH}$ from 
$60.0 \pm 10.0$ to $13.3 \pm 5.8$ on the VAS $(\mathrm{p}<0.05)$ and from $5.0 \pm 0$ to $1.3 \pm 0.5$ on the VSPI $(\mathrm{p}<0.05)$. Adverse effects were not identified.

NOO is a potent, non-sedative, quickly reversible and safe inhalative analgesic which reduces ISP considerably in patients receiving BT therapy for axillary and palmar HH thus substantially improving compliance of BT therapy.

\section{Botulinum toxin therapy: reduction of injection site pain by $\mathbf{p H}$-normalisation}

Dressler D. ${ }^{1,2}$, Adib Saberi F. ${ }^{1,2}$, Bigalke H. ${ }^{1,2}$

${ }^{1}$ Hannover Medical School, Movement Disorders Section, Department of Neurology, Hannover, Germany, ${ }^{2}$ Toxogen GmbH, Hannover, Germany

Botulinum toxin (BT) is injected intramuscularily and may produce injection site pain (ISP). We wanted to explore whether the $\mathrm{pH}$ value of the reconstituted BT drug contributes to ISP and, if so, what strategies can be applied to reduce it.

In part 1 of the study, $\mathrm{pH}$ values of different reconstitution solutions and of major BT drugs reconstituted with different reconstitution solutions were assessed. In part 2, the effects of reconstitution with normal saline (NS) and Ringer acetate (RA) were compared intraindividually and in a double blind fashion in 34 patients with blepharospasm.

$\mathrm{pH}$ values of reconstitution solutions were $5.52 \pm 0.02$ for $\mathrm{NS}$, 6.98 for RA, 6.31 for Ringer lactate, 6.56 for electrolyte and 5.31 for bacteriostatic solution. $\mathrm{pH}$ values for NS-reconstitution were $6.09 \pm 0.20$ for Botox ${ }^{\circledR}, 5.95 \pm 0.24$ for Dysport $^{\circledR}$ and $5.81 \pm 0.18$ for Xeomin ${ }^{\circledR}$. pH values for NS-reconstitution were $6.09 \pm 0.20$ for Botox ${ }^{\circledR}, 5.95 \pm 0.24$ for Dysport ${ }^{\circledR}$ and $5.81 \pm 0.18$ for Xeomin ${ }^{\circledR}$. pH values for RA-reconstitution were $6.95 \pm 0.03$ for Botox ${ }^{\circledR}$, $7.01 \pm 0.02$ for Dysport ${ }^{\circledR}$ and $6.87 \pm 0.06$ for Xeomin ${ }^{\circledR}$. By using RA instead of NS the pH could be increased by 0.86 for Botox ${ }^{\circledR}$, by 1.06 for Dysport ${ }^{\circledR}$ and by 1.06 for Xeomin ${ }^{\circledR} .47 \%$ of the patients experienced less ISP when Botox ${ }^{\circledR}$-RA was given rather than Botox ${ }^{\circledR}$ NS, $76 \%$ when Xeomin ${ }^{\circledR}$-RA was given rather than Xeomin ${ }^{\circledR}$-NS. None of the patients reported a difference in efficacy between NSand RA-reconstitution

Despite previous reports, reconstituted BT type A drugs show acidic $\mathrm{pH}$ values. Normalising these $\mathrm{pH}$ values by use of RA instead of NS reduces ISP considerably without sacrificing clinical efficacy.

\section{Reconstituting botulinum toxin drugs: shaking, stirring of what?}

Dressler D. ${ }^{1,2}$, Bigalke H. ${ }^{1,2}$

${ }^{1}$ Hannover Medical School, Movement Disorders Section, Department of Neurology, Hannover, Germany, ${ }^{2}$ Toxogen GmbH, Hannover, Germany

Most botulinum toxin (BT) drugs are stored as powders which need to be reconstituted with normal saline before clinical use. As botulinum neurotoxin (BNT), the therapeutically active ingredient, is a large doublestranded protein the process of reconstitution should be performed with special attention to mechanical stress applied. We wanted to test the mechanical stability of BNT during the reconstitution process.
For this, $100 \mathrm{MU}$ onabotulinumtoxinA (Botox ${ }^{\circledR}$, Irvine, CA, USA) was reconstituted with $2.0 \mathrm{ml}$ of $\mathrm{NaCl} / \mathrm{H} 2 \mathrm{O}$. Gentle reconstitution (GR) was performed with a $5 \mathrm{ml}$ syringe, a $0.90 \times 70 \mathrm{~mm}$ injection needle, 1 cycle of injection-aspiration-injection and 2 gentle shakes of the vial. Aggressive reconstitution (AR) was performed with a $5 \mathrm{ml}$ syringe, a $0.40 \times 40 \mathrm{~mm}$ injection needle, 10 injection-aspirationinjection cycles and $30 \mathrm{~s}$ of continuous shaking of the vial.

$\mathrm{AR}$ increased the time to paralysis in the mouse hemidiaphragm assay (HDA) from $72.0 \pm 4.6 \mathrm{~min}$ to $106.0 \pm 16.0 \mathrm{~min}(* \mathrm{p}=0.002$, two-tailed t-test after Kolmogorov-Smirnova test with Lilliefors correction for normal distribution). Construction of a calibration curve revealed that the increase in the time to paralysis was correlated with a loss of potency of from $100 \mathrm{MU}$ to $58 \mathrm{MU}(-42 \%)$.

BT users should use large diameter injection needles for reconstitution, apply two or three injection-aspiration-injection cycles and, maybe, shake the vials a few times to rinse the entire glass wall. Aggressive reconstitution with small diameter needles, prolonged injection-aspiration-injection and violent shaking should be avoided.

\section{Frontalis suspension surgery to treat patients with blepharospasm and eyelid opening apraxia: long- term results}

$\frac{\text { Dressler D. }}{\mathrm{R}^{2}}{ }^{2}$, Karapantzou C. ${ }^{2}$, Rohrbach S. ${ }^{2}$, Schneider S. ${ }^{2}$, Laskawi

${ }^{1}$ Hannover Medical School, Movement Disorders Section, Department of Neurology, Hannover, Germany, ${ }^{2}$ Department of Ear, Nose and Throat, Georg-August University, Göttingen, Germany

Blepharospasm may be accompanied by eyelid opening apraxia (EOA) reducing the efficacy of botulinum toxin (BT) therapy. The frontalis suspension operation (FSO) is then the only effective treatment option available. We want to report first long-term results with FSO.

We studied 15 patients with blepharospasm and EAO unresponsive to BT therapy ( 9 females, 6 males, age $61.9 \pm 11.5$ years). FSO was performed by applying 2 polytetrafluorethylen threads (PTFE, Gore-Tex ${ }^{\circledR}$ ) per eye connecting the frontalis muscle to the upper eye lid. Tension of the 2 carrés was set to produce a palpebral fissure width of $2-3 \mathrm{~mm}$. Therapy outcome was monitored by a Quality of Life Questionnaire (QoL-Q) and a Self-Assessment Calendar reviewing postoperative days 0-9 (T1), 10-89 (T2), 90-179 (T3), 180-365 (T4) and $>365$ days (T5).

Altogether 40 FSO were performed. Postoperatively, all patients reported improved eyelid opening, $4(27 \%)$ complete remission of symptoms. At $\mathrm{T} 1$ this improvement was $74.6 \pm 26.4 \%$ on the selfassessment scale, after 1 year $68.2 \pm 27.5 \%$. Throughout the observation period $(\mathrm{T} 1-\mathrm{T} 5)$ the improvement was $71.9 \pm 25.6 \%$. All 19 items on the QoL-Q (except for presence of involuntary eye lid closure) showed postoperative improvement. Adverse effects included circumscribed upper eyelid haematomas, suture extrusion, suture granuloma, lacrimation and infections. In all patients BT therapy had to be continued to treat orbicularis oculi contractions.

Our first long-term results demonstrate that FSO is a benign procedure producing robust and stable therapeutic effects on EOA in blepharospasm.

\section{Tetanus presented as hemiparesis and facial dystonia: 1 year follow up}

Dulski J. ${ }^{1}$, Schinwelski M. ${ }^{1,2}$, Sławek J. ${ }^{1,2}$ 
${ }^{1}$ St Adalbert Hospital Copernicus PL, Gdansk, Poland, Neurology Department, Gdansk, Poland, ${ }^{2}$ Medical University of Gdansk, Gdansk, Poland, Neurological and Psychiatric Nursing Department, Gdansk, Poland

Introduction: We report a case of tetanus presenting as the facial dystonia and hemiparesis with complete recovery and one-year video accompanied follow-up.

Methods: A 78-year old male, who sustained an abrasion to his right forearm, presented with dysarthria, dysphagia, prominent involuntary painful muscle spasms in the face, blepharospasm, risus sardonicus, trismus (lockjaw), neck muscle rigidity and mild weakness of the distal right upper extremity. The combined treatment of human antitetanus immunoglobulin, tetanus vaccine, penicillin, metronidazole and diazepam was given with no effect. He continued to worsen and due to the risk of the respiratory failure (finally not developed) he was sent to the Intensive Care Unit. The combined treatment was continued and his symptoms started to improve within 2 weeks. As the muscle rigidity has been improving, the patient developed significant right hemiparesis. At the follow-up examination 10 months after the accident the patient presented only slightly impaired dexterity of the right hand.

Discussion and conclusions: There are many reports of unusual presentations of tetanus, however, hemiparesis is uncommon, and to our knowledge, only one case was reported in the literature. We highlight the variability of clinical presentation of tetanus, the need to consider tetanus in cases of rapidly evolving muscle spasms following peripheral injuries and provide the guidelines on tetanus prophylaxis.

\section{The occurrence of lateral shift in idiopathic cervical dystonia}

Esposito M. ${ }^{1}$, Peluso S. ${ }^{1}$, Dubbioso R. ${ }^{1}$, Allocca R. ${ }^{1}$, Iorillo F. ${ }^{1}$, Coppola A. , Santoro L. ${ }^{1}$

${ }^{1}$ Federico II University of Naples, Department of Neurosciences, Reproductive Science and dentistry, Naples, Italy

Introduction: lateral shift (LS) of neck is an uncommon phenotype of idiopathic cervical dystonia (CD). It seems to be an unusual presentation of $\mathrm{CD}$ at onset and may be a phenotype that develops during disease course and treatment with botulinum toxin (BTX). Objective of this study is to find clinical aspects and details of the treatment with BTX that can influence the occurrence LS.

Methods: demographic and clinical data and modalities of the treatment with BTX of 38 consecutive patients with idiopathic CD were analyzed on a retrospective study. Two groups were identified on the presence of LS (LS group) and the lack of LS (nLS group).

Results: both groups of patients presented comparable demographic and clinical data. Actually there were no significant differences of disease and BTX treatment duration and of CD severity. The total amount of BTX injected was also comparable but the itereside difference of dose was significantly higher in the LS group (192 \pm 17.4 U vs $87,3 \pm 17.9 \mathrm{U}$ abo-BTX A; $\mathrm{p}<0.001)$. None of the patients of the LS group presented neck shift at onset of CD.

Conclusions: LS may not be an original phenotype of idiopathic CD and it could result from an imbalance of cervical muscle strength caused by treatments with BTX prevailing on one side. LS can be considered an effect of chronic therapy with BTX in CD with very asymmetrical muscle contractions.
Surgical replacement of Implantable pulse generators in deep brain stimulation: adverse events and risk factors in a multicenter cohort

Fytagoridis A. ${ }^{1,2}$, Jiltsova E. ${ }^{3}$, Heard T. ${ }^{4}$, Samuelsson J. ${ }^{2}$, Zsigmond $\mathrm{P}^{5}$, Skyrman S.

${ }^{1}$ Karolinska Institute, Department of Clinical Neuroscience, Neurosurgery, Stockholm, Sweden, ${ }^{2}$ Umeå University, Department of Pharmacology and Clinical Neuroscience, Umea, Sweden,

${ }^{3}$ Uppsala University Hospital, Department of Neurosurgery, Uppsala, Sweden, ${ }^{4}$ University of Queensland, Asia-Pacific Centre of Neuromodulation, Brisbane, Australia, ${ }^{5}$ Linköping University Hospital, Department of Neurosurgery, Linköping, Sweden

Background: Deep brain stimulation (DBS) is a growing treatment modality and most DBS systems require replacement of the implantable pulse generator (IPG) every few years. The literature is rather scarce regarding the potential impact of the adverse events of IPG replacements on the longevity of DBS treatments.

Objectives: To investigate the incidence of adverse events and postoperative infections of IPG replacements in a multicenter cohort.

Methods: Medical records of 808 patients from one Australian and five Swedish DBS centers with a total of 1293 IPG replacements were audited. A logistic regression model was used to ascertain the influence of possible predictors on the incidence of adverse events.

Results: The overall incidence of major infections was $2.3 \%$ per procedure, $3.7 \%$ per patient and $1.7 \%$ per replaced IPG. $93.3 \%$ of these patients needed partial or complete removal. DBS removal became permanent in 7 cases. There were statistical significant increased risk of infection for males (OR 3.6, $p=0.026)$ and for each previous IPG replacement (OR 1.6, $p<0.005)$.

Conclusions: The risk of postoperative infections of IPG replacements in DBS increases with each previous procedure and there is need to develop strategies to reduce the frequency of IPG replacements.

\section{Bilateral pallidal deep brain stimulation for dystonia: clinical outcome and electrode location}

Pérez Sánchez J.R. ${ }^{1}$, Mateo Sierra O. ${ }^{2}$, Muñoz González A. ${ }^{1}$, De La Casa Fages B. ${ }^{1}$, Domínguez Rubio R. ${ }^{1}$, González Quarante L.H. ${ }^{2}$, Garbizu J.M. ${ }^{2}$, Fernández García P. ${ }^{3}$, Guzmán de Villoria Lebiedziejeswki J. ${ }^{3}$, Fernández Carballal C. ${ }^{2}$, Grandas F. ${ }^{1}$

${ }^{1}$ Hospital Universitario Gregorio Marañón, Movement Disorders Unit, Madrid, Spain, ${ }^{2}$ Hospital Universitario Gregorio Marañón, Neurosurgery, Madrid, Spain, ${ }^{3}$ Hospital Universitario Gregorio Marañón, Radiology, Madrid, Spain

Introduction: Deep brain stimulation (DBS) of the globus pallidus internus (GPi) is a safe and effective treatment for generalized and segmental primary dystonia. There is comparatively less experience about the effects of GPi-DBS on acquired dystonia and only a few series analyze the electrode location after surgery. The objective of this study is to analyze the clinical outcome of patients with dystonia of different etiologies after GPiDBS and correlate it with the final electrode location.

Methods: We studied patients with dystonia treated with bilateral GPi-DBS at our institution and currently followed. Severity of dystonia was assessed by the Burke-Fahn-Marsden Dystonia Rating 
Scale (BFMDRS) and the Toronto Western Spasmodic Torticollis Rating Scale (TWSTRS) before and after surgery. The stimulation parameters including total electrical energy delivered $\left(\right.$ TEED $\left._{1 \mathrm{sec}}\right)$ were recorded. Two neuroradiologists blinded for outcome analyzed the anatomical location of electrodes in magnetic resonance imaging which was compared with the preoperative target planning.

Results and discussion: 17 dystonic patients were included: 9 primary (4 generalized and 6 segmental cranio-cervical dystonia), 7 secondary dystonia of different etiologies ( 2 cerebral palsy, 4 tardive and 1 pantothenate kinase-associated neurodegeneration). Mean follow-up was 59 months. 14 patients were responders with mean improvement of $61.5 \%$ in BFMDRS and $53.5 \%$ in TWSTRS. Three patients did not improve ( 1 cerebral palsy, 1 tardive and 1 idiopathic segmental). TEED $_{1 \text { sec }}$ was $132.3 \mathrm{~J}$ in responders and $184.9 \mathrm{~J}$ in non-responders. Precise location of electrodes was available in 11 patients ( 22 electrodes). Maximal deviation from the theoretical target in the axial plane was $2 \mathrm{~mm}$ in one electrode of 4 patients: 2 responders and 2 non-responders. In conclusion, bilateral pallidal deep brain stimulation is an effective therapy for dystonia of different etiologies. Deviations from the planned target $(2 \mathrm{~mm})$ in one electrode may originate a worse outcome despite the increase in TEED ${ }_{1 \mathrm{sec}}$.

\section{New presentation of Dopa Responsive Dystonia (DRD): Limb atrophy and muscle weakness}

\author{
Habibi S.A.H. ${ }^{1}$, Omrani F. ${ }^{1}$, Albanese A. ${ }^{2}$, Elia A.E. ${ }^{3}$ \\ ${ }^{I}$ Iran University of Medical Science, Department of Neurology, Rasool \\ Akram Hospital, Tehran, Iran, Islamic Republic of, ${ }^{2}$ Catholic \\ University, Carlo Besta National Neurological Institute, Department \\ of Neurology, Milan, Italy, ${ }^{3}$ Istituto Neurologico Carlo Besta, Milan, \\ Italy
}

Dopa Responsive Dystonia (DRD) is characterized by childhood dystonia with diurnal fluctuations and dramatic and sustained response to levodopa. Occasionally DRD is presented with other features such as tremor or Parkinsonism.

This case report introduces Limb atrophy and muscle weakness as a new presentation of DRD.

This case appeared to have combined neuropathy and weakness as the presenting feature and weakness and atrophy dominated the clinical picture.

\section{Adult onset dystonic tics: evidence for cerebellar involvement?}

Hidding U. ${ }^{1}$, Gelderbom M. ${ }^{1}$, Ganos C. ${ }^{1}$

${ }^{1}$ Universitätsklinik Hamburg Eppendorf, Hamburg, Germany

Introduction: Adult-onset dystonic tics are a rare entity. To date only a single case-series describing the clinical characteristics of 11 patients has been reported. Here, we describe a further patient with an unusual clinical syndrome with cerebellar signs and adult-onset dystonic tics.

Methods: Clinical and paraclinical details, alongside a video-documentation of the movement disorder are provided.

Results: A 56-year-old gentleman presented in our movement disorders clinic for clinical assessment and therapeutic evaluation. At the age of 44 he developed hyperkinesias involving the craniocervical region, as well as his voice and arms. In addition, he reported breathing irregularities. He also reported that since early childhood he had difficulties with balance, particularly with his left hand. There was no childhood history of tics. The majority of the movements occurred unintentionally. However he was able to control the hyperkinesias on demand, which led to an increasing sensation of internal tension. Releasing the hyperkinesias provided a relief from the unpleasant sensation. There was no change in the phenomenology or severity of hyperkinesias over the years. There was no relevant family history. Clinical examination (video) revealed multiple dystonic tics, including breathing dystonia, and pseudoathetosis involving the left arm, as well as left sided hemiataxia and spasticity. Laboratory examinations, including acanthocytes were normal. Prior testing for Huntington's disease was negative. Nerve conduction studies were also normal, but there were prolonged central latencies for somatosensory evoked potentials. Sequential brain MRIs over a period of 10 years showed a non-progressive in size pontomedullary malformation.

Conclusion: This case provides further evidence to the clinical phenotype of adult-onset dystonic tics. The unusual pontomedullary malformation and the cerebellar signs highlight a probable role of the brainstem and most likely cerebellum in dystonic tic pathophysiology.

\section{Bilateral pallidal deep brain stimulation for severe $\mathrm{X}$-linked dystonia-Parkinsonism (Lubag's disease): A plea for early intervention and potential programming strategy}

\author{
Sandhu M. ${ }^{1}$, Poologaindran A. ${ }^{1}$, Polyhronopoulos N. ${ }^{1}$, Honey C.R. ${ }^{1}$ \\ ${ }^{I}$ The University of British Columbia, Division of Neurosurgery, \\ Vancouver, Canada
}

Introduction: XDP (also known as Lubag's disease) is a rapidly progressive neurodegenerative movement disorder featuring symptoms of dystonia and Parkinsonism. To date, seven cases of XDP have been treated with GPi DBS with a mean pre-operative score of 63/120 on the BFMDRS. Here, we report the most severe case ever of XDP treated with GPi DBS and highlight a potential programming strategy and plea for early intervention.

Methods: A 34-year-old male with family origins from Panay, Philippines developed spontaneous cervical dystonia that was associated with blepharospasm, difficulty opening/closing the jaw, and dysarthria. After failing medical management and genetic testing strongly implicating XDP, the patient immediately underwent GPi DBS under general anesthesia. Pre-operatively, the patient scored $108 / 120$ on the BFMDRS and 20/20 on the MDS.

Results: At the 6-month follow-up, the patient had a remarkable early improvement as measured by the BFMDRS and MDS: 108-11/120 and $20-3 / 20$ respectively. The patient went from being wheel chairbound and using a gastrostomy tube to playing sports and eating normally, but with some residual blepharospasm and dysarthria. Video 1 on iPad highlights pre- and post-operative $(6 \mathrm{mo}$.) status of the patient. Final stimulation parameters at 6 months were Left$\mathrm{C}+0-, 3.0 \mathrm{~V}, 90 \mu \mathrm{s}, 130 \mathrm{~Hz}$ and Right $-\mathrm{C}+8-, 4.2 \mathrm{~V}, 90 \mu \mathrm{s}$, $130 \mathrm{~Hz}$.

Discussion: GPi DBS was well-tolerated in this very severe case of XDP. Despite XDP's classification as a dystonia, our group and others found that monopolar, not bipolar, stimulation settings resulted in the most improvement. This programming strategy is worth testing first before bipolar stimulation. Unfortunately, the disease appeared to rapidly progress at the 1-year follow-up (20/120 on BFMDRS). We highly recommend neurosurgical intervention to be considered immediately after the time of diagnosis in order to widen the therapeutic window DBS offers. 


\section{A prospective, randomized, blinded assessment of multi-target deep brain stimulation for hemi- dystonia: Targeting both cerebellar and pallidal circuitry}

Ivanishvili Z. ${ }^{1}$, Poologaindran A. ${ }^{1}$, Sandhu M. ${ }^{2}$, Polyhronopoulos N. ${ }^{1}$, Honey C.R. ${ }^{1}$

${ }^{I}$ The University of British Columbia, Division of Neurosurgery, Vancouver, Canada, ${ }^{2}$ The University of British Columbia, Vancouver, Canada

Introduction: Dystonia is increasingly being interpreted as a multinodal "network" disorder involving both cerebellar and pallidal circuitry. In hemi-dystonia patients who are poor or non-responders to GPi DBS, we aimed to investigate multi-target GPi and Vim DBS versus each target alone in a prospective, randomized, double-blinded manner. We wish to present preliminary results of $n=2$ of 6 patients who have completed this study.

Methods: DBS leads were implanted in the contralateral GPi and Vim for hemi-dystonia. Patients were randomized to either GPi, Vim, or multi-target $(\mathrm{GPi}+\mathrm{Vim})$ stimulation for 3 months at a time. At the end of each 3-month trial period, patients were evaluated in a double-blinded manner using the BFMDRS. After 9 months of blinded stimulation, the best setting was evaluated unblinded for 6 months.

Results: Multi-target $(\mathrm{GPi}+\mathrm{Vim})$ stimulation was clinically the most effective treatment and resulted in the most improvement in function and quality of life. The patients' hemi-dystonia improved by $50 \%$ on the BFMDRS in the multi-target stimulation trial period and at the 6-month follow-up. The patient's quality of life significantly improved and multi-target stimulation was the patients' preferred setting.

Discussion: In addition to traditionally-viewed pallidal dysfunction in dystonia, recent evidence indicates cerebellar dysfunction as well. While only two patients, treating both cerebellar and pallidal circuits appears more effective than treating a single circuit in hemi-dystonia. Given the small sample size, caution should be applied in generalizing these preliminary results. It is important to appreciate that hemi-dystonia patients are typically less functionally impaired (on BFMDRS) compared to patients with primary dystonia. Thus, their degree of improvement after surgery may appear more modest and not as dramatic. Multi-target DBS should be further explored in dystonia patients with limited or no response to GPi DBS.

\section{Bilateral pallidotomy for cervical dystonia after failed selective peripheral denervation}

\author{
Horisawa S. ${ }^{1}$, Kawamata T. ${ }^{1}$, Taira T. ${ }^{1}$ \\ ${ }^{1}$ Tokyo Women's Medical University's Medical University, Shinjyuku, \\ Japan
}

Background: We investigated the utility of pallidotomy for cervical dystonia following failed selective peripheral denervation.

Case description: A 36-year-old man presented with cervical dystonia with limited range of motion of the left shoulder, particularly in abduction. His Tsui score was 8. Due to the ineffectiveness of botulinum toxin injection, he underwent selective peripheral denervation with an unsatisfactory outcome, with a postoperative Tsui score of 6 . Simultaneous bilateral pallidotomy was performed with local anesthesia at 1 year after the initial surgery. The day after the pallidotomy, all dystonic symptoms were markedly improved, except for the limited range of shoulder abduction. The Tsui score was recorded as 1 at 1 week after the pallidotomy. Transient aggressive behavior was the only postoperative complication in the present case. During clinical follow-up period of 1 year, no recurrence of the symptoms was observed.

Conclusions: Pallidotomy represents a feasible and efficacious treatment for cervical dystonia refractory to SPD without hardware related complications.

\section{Stereotactic thalamotomy for the yips (sports-related dystonia)}

Horisawa S. ${ }^{1}$, Kawamata T. ${ }^{1}$, Taira T. ${ }^{1}$

${ }^{1}$ Tokyo Women's Medical University's Medical University, Shinjyuku, Japan

Movement-specific involuntary movements that occur in athletes are often called the yips. Although the underlying cause of the yips is still controversial, it is broadly divided into the psychogenic origin (choking) or neurological origin (task-specific dystonia). We herein report two athletes with task-specific dystonia who diagnosed with yips treated by stereotactic thalamotomy that resulted in substantial success. Among patients diagnosed with yips, some can benefit from stereotactic thalamotomy based on a concept of task-specific dystonia.

\section{Bilateral stereotactic thalamotomy for bilateral musician's hand dystonia}

\author{
Horisawa S. ${ }^{1}$, Kawamata T. ${ }^{1}$, Taira T. ${ }^{1}$ \\ ${ }^{1}$ Tokyo Women's Medical University's Medical University, Shinjyuku, \\ Japan
}

Focal hand dystonia in musician, also called as musician's dystonia, is a task-specific movement disorder characterized by unwanted involuntary muscle contraction occurring only when playing a musical instrument. We have already reported marked improvement of medically intractable unilateral musician's hand dystonia by stereotactic unilateral thalamotomy. Its procedure can provide immediate and long-standing effect. We herein treated two patients with bilateral musician's hand dystonia by staged bilateral stereotactic thalamotomy and they experienced marked improvement of bilateral symptoms immediately after the surgery. Both patients became to play their musical instruments at the same level of musical skill as before the onset of dystonia. Transient dysarthria and hypophonia developed but these symptoms didn't impair their activities of daily living. No recurrence of symptoms has been observed for over a year after each second surgery. These outcomes indicated stereotactic thalamotomy also can be useful for medically intractable musician's dystonia in both hands. This is the first report about bilateral musician's hand dystonia treated by bilateral stereotactic thalamotomy.

\section{Unilateral pallidotomy for cervical dystonia}

\author{
Horisawa S. ${ }^{1}$, Kawamata T. ${ }^{1}$, Taira T. ${ }^{1}$ \\ ${ }^{I}$ Tokyo Women's Medical University's Medical University, Shinjyuku, \\ Japan
}

Objective: We investigated the validity of unilateral pallidotomy for cervical dystonia using severity scale of Toronto Western Spasmodic Torticollis Rating Scale (TWSTRS, range 0-35). 
Background: Although stereotactic neurosurgery for cervical dystonia means basically the same procedure as bilateral deep brain stimulation (DBS) of globus pallidus internus (GPi), lesioning of GPi (pallidotomy) for cervical dystonia is rarely reported. Furthermore, some reports showed efficacy of unilateral pallidal stimulation for cervical dystonia. However, efficacy of unilateral pallidotomy for cervical dystonia has not been investigated.

Methods: 6 patients ( 4 men/2 women) with cervical dystonia underwent unilateral pallidotomy. We used severity scale of TWSTRS as evaluation procedures before and 1 week after the surgery.

Results: The mean age at onset and surgery were 34.7 (SD 15.3) and 47.2 (SD 11.4). The mean preoperative severity scale of TWSTRS were 19.2(SD 4.8) and 4.7 (SD 3.9) (75.5\% reduction). No perioperative adverse event was observed.

Conclusions: Albeit short observational period, this study suggest unilateral pallidotomy is safe and effective procedure for cervical dystonia.

\section{Objective evaluation of performance stress in musicians with focal hand dystonia: A case series}

\author{
Ioannou C.I. ${ }^{1}$, Furuya S. ${ }^{2}$, Altenmüller E. ${ }^{1}$
}

${ }^{1}$ Hanover University of Music, Drama and Media, Institute of Music Physiology and Musicians Medicine, Hannover, Germany, ${ }^{2}$ Sophia University, Department of Information and Communication Science, Tokyo, Japan

Five musicians suffering from focal dystonia participated in a pilot study which examined the feasibility of an experimental protocol designed to assess musicians' motor performance under stress. Electrocardiography (ECG), free cortisol levels and subjective assessments were used to monitor alterations of the hypothalamic-pituitary-adrenal (HPA) axis. As measures of motor outcome, temporal variability of finger movements and muscular co-contraction (EMG) of the wrist flexor and extensor were assessed. Findings suggest that the specific experimental design could be successfully applied. Several methodological issues such as carryover effects, the use of free cortisol, the inclusion of a double baseline and the classification of dystonic patients into stress responders and non-responders are analyzed and discussed.

\section{The impact of stress on motor performance in skilled musicians suffering from focal dystonia: Physiological and psychological characteristics}

\author{
Ioannou C.I. ${ }^{1}$, Furuya S. ${ }^{2}$, Altenmüller E. ${ }^{1}$ \\ ${ }^{1}$ Hanover University of Music, Drama and Media, Institute of Music \\ Physiology and Musicians' Medicine, Hanover, Germany, ${ }^{2}$ Sophia \\ University, Department of Information and Communication Science, \\ Tokyo, Japan
}

Several studies have shown that motor functions can be altered under stressful conditions. However, the impact of stress on motor performance of musicians suffering from focal dystonia (FDM) remains largely unknown. The current study assessed motor performance in 20 FDM patients and 16 healthy musicians (HM) before and under stress. Stress was manipulated using the Trier Social Stress Test (TSST). Motor performance was evaluated during rapid scale playing based on analysis of the electromyographic (EMG) activity (mean activity and co-contraction of the wrist flexor/extensor) and temporal variability (fine-motor control), while electrocardiography (ECG) and the level of free cortisol were used to test for objective alterations of the hypothalamic-pituitaryadrenal (HPA) axis. Finally, the psychological profiles (based on anxiety, perfectionistic and stress coping features) of both groups were analyzed using psycho-diagnostic standardized questionnaires. Results showed that patients' motor impairments did not change under acute stressful conditions. However, an increase in muscular co-contractions was observed, reflecting a "normal" physiological muscular reaction under stressful conditions. Psycho-diagnostic analysis revealed higher levels of anxiety, stress and perfectionism in $40 \%$ of the patients. Although the motor behavior between these patients and patients with an opposing psychological profile did not differ, interestingly patients characterized by stressful and perfectionistic personalities had, on average, developed dystonia about 10 years earlier than the rest of the patients. The current study suggests that acute stress conditions may not have any direct impact on fine motor control of FDM patients. However psychological traits associated with increased stress, anxiety and perfectionism may have a long-lasting effect on the motor function of affected musicians, by promoting the acceleration or even the triggering of dystonia.

\section{Dystonia, tremor, and dystonic tremor}

\author{
Rosen A. ${ }^{1}$, Shaikh A. ${ }^{2}$, Jinnah H.A. ${ }^{1}$ \\ ${ }^{I}$ Emory University, Neurology, Atlanta, GA, USA, ${ }^{2}$ Case Western \\ Reserve University, Cleveland, $\mathrm{OH}, \mathrm{USA}$
}

Introduction: Dystonia is a disorder with sustained or intermittent muscle contractions causing abnormal, often repetitive and patterned movements or postures. In contrast, tremor is a disorder with regular and rhythmical oscillation of a body region. While dystonia and tremor are viewed as distinct disorders, they have close epidemiological and phenomenological relationships and occur together frequently. The goal was to provide guidance for debates regarding the nosology of tremor in dystonia.

Methods: Subjects were recruited through 38 Dystonia Coalition sites in North America, Europe and Australia. Subjects were at least 18 years of age and had a diagnosis of isolated dystonia. Any region of the body could be affected, alone or in various combinations. As part of an ongoing natural history study, all sites collected standardized clinical data, including an exam that detailed presence or absence of dystonia and tremor in each body part, type of tremor seen, and whether tremor was the dominant clinical feature.

Results and discussion: 2301 subjects were included in the analyses. In keeping with previous reports, a large proportion of dystonia subjects had tremor on exam, with co-prevalence varying according to body region affected, how dystonic tremor is defined, and variations in enrolling sites. There are three hypothetical models to explain the observed relationships between tremor and dystonia. (1) Tremor and dystonia are biologically unrelated disorders, but a movement disorder indistinguishable from isolated tremor may sometimes occur in dystonia. (2) Tremor and dystonia are caused by the same biological process, with the overt clinical manifestations being pleiomorphic consequences falling at different ends of a continuous spectrum. (3) Tremor and dystonia are caused by different biological processes, but they frequently overlap because the biological pathways are partially related. Current definitions imply they are mutually exclusive disorders. Identifying their biological bases may clarify which model best represents their relationship. 


\section{Underlying heredity is associated with alcohol responsiveness in dystonia}

Junker J. ${ }^{1}$, Brandt V. ${ }^{1}$, Weissbach A. ${ }^{1}$, Lohmann K. ${ }^{1}$, Perlmutter J.S. ${ }^{2}$, Wright L.J. ${ }^{2}$, Rosen A.R. ${ }^{3}$, Klein C. ${ }^{1}$, Jinnah H.A. ${ }^{3}$, Brueggemann

N. ${ }^{1}$, Dystonia Coalition Investigators, Dystonia Genetic Consortium

${ }^{1}$ University of Luebeck, Institute of Neurogenetics, Luebeck, Germany, ${ }^{2}$ Washington University in St. Louis, Department of Neurology, St. Louis, MO, USA, ${ }^{3}$ Emory University, Department of Neurology, Atlanta, GA, USA

Introduction: Some dystonia patients show a remarkable improvement of their dystonia due to alcohol use. It remains elusive whether alcohol responsiveness is associated with the presence of (a) a positive family history, (b) dystonic tremor, or (c) a sensory trick.

Objective: To determine predictors of alcohol responsiveness in a large cohort of dystonia patients (Dystonia Coalition Cohort).

Methods: Data from 920 patients with isolated dystonia (mean age: $60.0 \pm 11.9$ years, female $\mathrm{n}=650$ ) and no evidence of a psychogenic origin of the disease were included. Patients answered a standardized questionnaire and were clinically examined using a standardized video protocol.

Results: Alcohol responsiveness was not related to gender $(70.1 \%$ female in the responsive vs. $70.9 \%$ in the non-responsive group, $\mathrm{p}=0.828$ ) or severity of dystonia (Burke Fahn Marsden motor score $7.9 \pm 9.0$ vs. $7.2 \pm 6.5, \mathrm{p}=0.587)$. Age at onset was lower in patients with alcohol responsiveness $(40.8 \pm 15.1$ vs. $47.4 \pm 14.1$ years, $\mathrm{p}=0.000)$. Alcohol responsiveness related to a positive family history for movement disorders $(\mathrm{p}=0.004)$, presence of tremor $(\mathrm{p}=0.001)$ and an effective sensory trick $(\mathrm{p}=0.029)$. Furthermore, alcohol responsiveness differed among dystonia subgroups (multifocal or generalized $>$ focal or segmental; cervical and laryngeal $>$ cranial and limb; oromandibular or lingual $>$ blepharospasm and Meige syndrome).

Discussion: The association of alcohol responsiveness and a positive family history for movement disorders suggests that dystonia patients with an underlying genetic contribution may have a more beneficial response to alcohol. Alcohol may specifically improve dystonic tremor, and the presence of a sensory trick may be a clinical predictor for alcohol responsiveness. Subgroup analyses suggest a caudo-rostral gradient with strongest response to alcohol in patients with more widespread dystonia and lowest improvement in blepharospasm, which is in keeping with an association of alcohol responsiveness and underlying heredity.

\section{Epidemiology of spastic dystonia in stroke patients: pilot results of SONAR study}

Dornak T. ${ }^{1}$, Justanova M. ${ }^{2}$, Kanovsky P. ${ }^{3}$, Otruba P. ${ }^{1}$, Bareš M. ${ }^{2}$, Jech R. ${ }^{4}$, Hoskovcova M. ${ }^{4}$, Navratilova D. ${ }^{1}$, Muzik J. ${ }^{5}$, Dusek L. ${ }^{5}$

${ }^{1}$ University Hospital Olomouc, Department of Neurology, Olomouc, Czech Republic, ${ }^{2}$ Faculty of Medicine, Masaryk University and St. Anne Teaching Hospital, Department of Neurology, Brno, Czech Republic, ${ }^{3}$ Palacky University Medical School, Department of Neurology, Olomouc, Czech Republic, ${ }^{4}$ First Faculty of Medicine, Charles University, Department of Neurology and Clinical Neuroscience, Prague, Czech Republic, ${ }^{5}$ Masaryk University in Brno, Institute of Biostatistics and Analyses, Brno, Czech Republic

Objective: The main aim of the study was a point and interval estimate of the incidence and prevalence of spasticity following ischemic stroke (IS) for the regional stroke centers in the Czech Republic; the secondary goal was to identify predictors for the development of spasticity.

Methods: In the prospective cohort study, 221 consecutive patients with clinical signs of central paresis due to a first-ever IS were examined in the acute stage. All patients met following criteria: patients with primary IS with carotid origin and neurological deficit present longer than 7 days after the stroke onset. The degree and pattern of paresis and muscle tone, the Barthel Index, baseline characteristic and demographic data were evaluated. Spasticity was assessed using modified Ashworth Scale (MAS) and defined as $>1$ MAS in any of the examined muscle groups.

Results: Out of 221 included patients (134 males; mean age $69.7 \pm 12.0), 93(42.1 \%)$ patients have developed spasticity during the first 10 day after stroke onset. Seventy-six (34.4\%) patients presented with mild neurological deficit (modified ranking scale 0-2) and $55(24.9 \%)$ patients were bedridden. Spasticity was noted in $42.1 \%$ patients with neurological deficit due to first-ever stroke in carotid territory during the first 10 days after stroke onset. However, severe spasticity was rare.

Funding: Supported by grant AZV MZ CR Nr. 15-31921A and by the grant of the Internal Grant Agency of Palacky University IGA UP LF2016-017.

\section{Changes in sensorimotor network activations after botulinum toxin injections in cervical dystonia}

\author{
Kanovsky P. ${ }^{1}$, Nevrly M. ${ }^{1}$, Hlustik P. ${ }^{1}$, Otruba P. ${ }^{1}$, Tudos Z. ${ }^{2}$, Hok P. ${ }^{1}$ \\ ${ }^{1}$ Palacky University Medical School, Department of Neurology, \\ Olomouc, Czech Republic, ${ }^{2}$ Palacky University Medical School, \\ Department of Radiology, Olomouc, Czech Republic
}

Objective: To advance the knowledge of focal dystonia pathophysiology and further explain the changes in central sensorimotor circuits induced by botulinum toxin injection to dystonic muscles.

Background: The pathophysiology of cervical dystonia (CD) and other focal dystonias has not yet been fully explained. Results from neurophysiological and morphological studies suggest significant involvement of the basal ganglia, thalamus and cerebellum; nevertheless, functional abnormalities in premotor and primary sensorimotor cortical areas are considered a crucial factor in the development of focal dystonia. Botulinum toxin A (BoNT-A) injections are the most effective, although symptomatic, treatment of $\mathrm{CD}$. Recent studies showed evidence of not only a direct effect of BoNT-A on the neuromuscular junction, but also remotely on the central sensorimotor circuits.

Design/Methods: Twelve patients with $\mathrm{CD}$ were examined with functional MRI during a skilled hand motor task before the first BoNT-A injection; the examination was repeated 4 weeks after the first BoNT-A injection to the dystonic neck muscles. Twelve age- and gender-matched healthy controls were examined using the same protocol without BoNT-A injection.

Results: Effective BoNT-A treatment led to reduced activation of the ipsilateral supplementary motor area and dorsal premotor cortex. The post-treatment sensorimotor maps showed significantly decreased basal ganglia activation. Before BoNT-A injection, activation maps were significantly different between the patient group and the control group, in contrast, results of the second examination after BoNT-A injection demonstrated no significant differences between the groups. 
Conclusion: Abnormalities in sensorimotor activation in cervical dystonia extend beyond circuits controlling the affected body part and its substantial change or "normalization" might be induced by BoNTA treatment.

Research supported by Institutional Support of Research Organization MZ CR RV- FNOL 2016.

\section{Multi target treatment of NBIA-related dystonia with subthalamic or pallidal nucleus deep brain stimulation}

\author{
$\underline{\text { Kmiec T. }}{ }^{1}$, Koziara H. ${ }^{2,3}$, Bonicki W. ${ }^{2,3}$, Brodacki B. ${ }^{4}$, Mandat T. ${ }^{2,3}$ \\ ${ }^{1}$ Institute Centrum Zdrowia Dziecka, Neurology, Warszawa, Poland, \\ ${ }^{2}$ Maria Sklodowska-Curie Oncology Memorial Center, Neurosurgery, \\ Warszawa, Poland, ${ }^{3}$ Institute of Psychiatry and Neurology, \\ Neurosurgery, Warszawa, Poland, ${ }^{4}$ Military Medical Institute, \\ Neurology, Warszawa, Poland
}

Conservative and surgical treatment of Neurodegeneration with Brain Iron Accumulation (NBIA) is difficult and frequently ineffective. The authors present a group of patients with clinically and radiologically diagnosed NBIA with genetically confirmed PANK2 mutation, treated with deep brain stimulation.

Materials and methods: Twelve patients with confirmed PANK2 mutation (NBIA-PKAN) were treated with deep brain stimulation between 2008 and 2015. Age of the patients varied from 8 to 24 years. The clinical condition of the patients was evaluated with scales and video recorded. At all cases the permanent electrodes were implanted to the subthalamic nuclei or globus pallidus. The surgical procedure was undertaken under general anesthesia. The target was identified with direct and indirect method. Intrasurgical macrostimulation and microrecording were used for neurophysiological evaluation of the target. Postsurgical local field potentials were recorded in all cases

Results: Neither neurological deterioration nor surgical complication were noted among the group. Caregivers of the patients noted subjective improvement of the clinical state of the subjects that was confirmed with tailored scales. More significant improvement was noted among STN group compared to GPi group.

Conclusions: Subthalamic or pallidal deep brain stimulation reduces dystonic movements among NBIA patients. The technique carries minimal surgical risk, and improves quality of life of the patients.

\section{Peripheral nerve injury leads to dystonia in DYT1 transgenic rats}

\author{
Knorr S. ${ }^{1}$, Grundmann-Hauser K. ${ }^{2}$, Volkmann J. ${ }^{1}$, Ip C.W. ${ }^{1}$ \\ ${ }^{1}$ University of Wuerzburg, Department of Neurology, Wuerzburg, \\ Germany, ${ }^{2}$ University Tuebingen, Institute of Medical Genetics \\ and Applied Genomics, Rare Disease Center Tuebingen, Tuebingen, \\ Germany
}

None of the existing non-neurodegenerative rodent models of DYT1 dystonia show the typical limb-onset dystonic phenotype seen in human. Based on the hypothesis that a "second hit" such as peripheral injury could elicit the dystonic phenotype in genetically predisposed subjects, we induced clinically apparent dystonia in a transgenic DYT1 rat model, that harbors the full human mutant Tor1A gene and does not show dystonia per se, by a sciatic nerve injury.
Development of dystonia of the affected limb was investigated by using a newly developed $0-5$ point scoring: 0 : no abnormal dystonic movement; 1: short hindlimb retraction and clenching of the foot/leg with episodes lasting $<3 \mathrm{~s}$ or $\leq 2$ repeats of hindlimb retraction and clenching of the foot/leg; 2 : hindlimb retraction and clenching of the foot/leg with episodes lasting $\geq 3 \mathrm{~s}$ or $\geq 3$ repeats of hindlimb retraction and clenching of the foot/leg; 3: hindlimb retraction and clenching of the foot/leg occurring for a total of $10 \mathrm{~s}$ to $<50 \%$ of the recorded time; 4: hindlimb retraction and clenching of the foot/leg lasting a total of $50 \%$ of the recorded time; 5: hindlimb retraction and clenching of the foot/leg for a total of $>50 \%$ of the recorded time. Dystonia duration and number of dystonic retractions of both hindlimbs were added for scoring.

Two weeks after nerve injury a maximum dystonia score of $\sim 5.0$ in wt and DYT1 rats was observed, followed by a slow decrease of dystonia in both genotypes. The dystonia score decreased in wt rats down to a minimum of $\sim 0.3$ on week 10 while DYT1 transgenic rats stayed at a higher level of about $\sim 2.0$ at the same time point. Dystonia after nerve injury in DYT1 transgenic rats can be revealed by this newly established clinical dystonia scoring system.

\section{Blepharospasm: Long-term treatment with either Botox ${ }^{\circledR}$, Dysport ${ }^{\circledR}$ or Xeomin ${ }^{\circledR}$}

Kollewe K. ${ }^{1}$, Mohammadi B. ${ }^{2}$, Köhler S. ${ }^{1}$, Pickenbrock H. ${ }^{1}$, Dengler R. , Dressler D. ${ }^{1}$

${ }^{1}$ Medical School Hannover, Neurology, Hannover, Germany,

${ }^{2}$ International Neuroscience Institute (INI), Hannover, Germany

Botulinum toxin (BT) therapy is the treatment of choice for blepharospasm (BPS). Currently available BT type A drugs include Botox, Dysport and Xeomin. Until now, there are few long-term studies on BT therapy for BPS. This is the first long-term study comparing all three major BT drugs. We collected treatment, efficacy and adverse effect data on BPS patients treated with either Botox, Dysport or Xeomin for at least 8 consecutive treatments. 288 patients ( 208 females, 80 males, age $62 \pm 12$ years) were included in this study. The treatment time was $11.2 \pm 4.1$ years covering 10701 injection series. Doses were $47 \pm 10 \mathrm{MU}$ for Botox, $120 \pm 35$ MU for Dysport and $62 \pm 11$ MU for Xeomin (Botox dose vs Xeomin dose: $\mathrm{p}<0.001$, unpaired t-test). $85 \%$ of all patients had stable doses. The onset of the therapeutic effect was after $6.1 \pm 3.3$ days and its duration lasted $10.2 \pm 3.5$ weeks. The Global Clinical Improvement $(\mathrm{GCI}, 0=$ no, $1=$ slight, $2=$ moderate, $3=$ marked improvement in severity and function) as estimated by the patient was $2.5 \pm 0.6$. It was stable in $90 \%$ of the patients. Adverse effect frequency was $3.0 \%$ (ptosis $2.3 \%$, dry eye $0.5 \%$, diplopia $0.2 \%$ ). None of these findings was significantly different between Botox, Dysport and Xeomin.

Our study, one of the largest studies on BT therapy of BPS and the study with the longest follow-up, confirm that BT therapy produces robust clinical improvement which is stable throughout the treatment time. Adverse effects were rare, mild and always transient. BT therapy is a safe and effective treatment for BSP. Shorter interinjection intervals may improve therapeutic results.

\section{A study of respondents to have musician's dystonia from a questionnaire survey performed in a music college}

Konaka K. ${ }^{1}$, Mihara M. ${ }^{1}$, Mochizuki H. ${ }^{1}$ 
${ }^{1}$ Osaka University Medical School of Graduate, Neurology, Suita, Japan

Background/objective: Musician's dystonia is a task specific dystonia. Though part of detail has been noticed, it is not widely informed. Our aim was to detect the prevalence and clarify the features.

Method: We conducted a questionnaire in two music colleges in purpose of extracting the prevalence and characteristics of musician's dystonia among music students. We explained the characteristics of musician's dystonia on the questionnaire sheet.

Results: There were 596 valid responses from 1468 college students. The average age of subjects was $19.8 \pm 2.8$ years old. There were 528 women and 68 men. There were 38 respondents answering to have musician's dystonia. According to previous descriptions, we finally made a decision whether their specific symptoms are adequate for musician's dystonia. There were 10 answers (which was $0.7 \%$ of all the answers) replying to have musician's dystonia. They were 5 pianists, 4 brass players and 1 woodwind player. They noticed their first symptom $9.1 \pm 4.7$ years after they started practicing the instruments. On the other hand, there were 28 respondents that we decided the symptoms do not satisfy the symptoms of musician's dystonia. Though the majority $(71.4 \%)$ of them contained information of stage fright or anxiety, those were less recognized in respondents of students judged to have musician's dystonia $(20.0 \%)$.

Conclusions: We ascertained the prevalence of musician's dystonia in students of a music college. Disorder of musical motor performance seems to be easily influenced by emotional stress, therefore there is a difficulty in distinguishing musician's dystonia.

\section{Meta-analytical and electrophysiological evidence for executive dysfunction in primary dystonia}

\author{
Lange F. ${ }^{1}$, Seer C. ${ }^{1}$, Salchow C. ${ }^{1}$, Dengler R. ${ }^{1}$, Dressler D. ${ }^{1}$, Kopp B. ${ }^{1}$ \\ ${ }^{1}$ Hannover Medical School, Department of Neurology, Hannover, \\ Germany
}

Impaired motor control in primary dystonia has been linked to cortico-basal ganglia alterations that may also give rise to changes in executive functioning. However, no conclusive evidence for executive dysfunction in patients with primary dystonia has been reported yet. We conducted a meta-analysis of the relationship between primary dystonia and performance on the Wisconsin Card Sorting Test (WCST), an established test of executive functioning. Its results revealed a significant effect of medium size, indicating that primary dystonia is associated with moderate performance deficits on the WCST. Building on this finding, we conducted an event-related potential (ERP) study to elucidate the cognitive and neural mechanisms underlying executive dysfunction in primary dystonia. Eighteen patients with blepharospasm, a common form of primary focal dystonia, and 34 healthy matched controls completed a computerized version of the WCST. We specifically compared indicators of two distinct components of executive functioning: set shifting and rule inference. On a behavioral level, blepharospasm patients seemed to have particular difficulty integrating information to infer the correct task rule. In addition, P3a amplitude (as an electrophysiological marker of rule-inference processes) was selectively attenuated in blepharospasm patients.
Executive dysfunction in blepharospasm can thus be attributed to a selective rule-inference deficit, whereas set-shifting abilities are largely unaffected by the disease. Moreover, P3a amplitude attenuation was related to disease duration, indicating that this ERP might serve as a neural indicator of disease progression and executive dysfunction in primary dystonia. These results demonstrate for the first time that pathophysiological alterations in primary dystonia might affect cortical activation for executive functioning.

\section{German registry of pediatric deep brain stimulation in patients with childhood-onset dystonia (GEPESTIM): Adverse events}

Koy A. ${ }^{1}$, Hellmich M. ${ }^{2}$, Kühn A.A. ${ }^{3}$, Krause P. ${ }^{3}$, Huebl J. ${ }^{3}$, Schneider G.-H. ${ }^{3}$, Weinsheimer M. ${ }^{1}$, Deuschl G. ${ }^{4}$, Erasmi R. ${ }^{4}$, Falk D. ${ }^{4}$, VisserVandewalle V. ${ }^{1}$, Pauls K.A.M. ${ }^{1}$, Schnitzler A. ${ }^{5}$, Wojtecki L. ${ }^{5}$, Vesper J. ${ }^{5}$, Korinthenberg R. ${ }^{6}$, Coenen V.A. ${ }^{6}$, Lütjens G. ${ }^{7}$, Krauss J.K. ${ }^{7}$,

Timmermann L. ${ }^{1}$, GEPESTIM study group

${ }^{1}$ University Hospital Cologne, Cologne, Germany, ${ }^{2}$ University of Cologne, Cologne, Germany, ${ }^{3}$ Charité University Medicine Berlin, Berlin, Germany,

${ }^{4}$ University Hospital of Schleswig-Holstein, Campus Kiel, Kiel, Germany, ${ }^{5}$ University Hospital of Düsseldorf, Düsseldorf, Germany, ${ }^{6}$ Freiburg University Medical Centre, Freiburg, Germany, ${ }^{7}$ Medical School, MHH, Hannover, Germany

Introduction: Data on adverse events (AEs) in pediatric patients undergoing deep brain stimulation (DBS) is limited, especially for long-term outcomes, because of small numbers in single center series and lack of multi-center trials.

Methods: A German registry on pediatric DBS (GEPESTIM) was created to collect data of patients with dystonia and DBS up to the age of 18 years. All postoperative AEs were documented.

Results: So far, data of 44 patients (mean age 12.8 years at time of implantation) have been included in the registry. 61 AEs were documented for 31 patients. IPG replacement due to battery expiry was the most common reason for surgical intervention. In 15 patients 20 IPGs had to be removed due to depleted batteries after an average time after last implantation of $3.7 \pm 1.8$ years. Infections and wound healing problems were other frequent indications for hardware removal or replacement. Reversible causes included fever, wound infection, and stimulation induced side-effects. Analysis of the (mean) number of AEs in relation to different age groups at time of surgery and time of follow-up revealed that patients aged 7-9 years at time of surgery had the highest number of AEs per patient (2.0) up to 4 years after implantation.

Discussion: The number of AEs associated with pediatric DBS seems similar to previous reports, but higher than in adult patients undergoing DBS. The high rate of infection might be attributed to severe motor impairment and undernourishment, which is commonly seen in pediatric patients suffering of acquired forms of dystonia. Therefore, the use of antibiotics should be reconsidered. In view of the frequent IPG replacements due to battery expiry, the implantation of rechargeable IPGs may be favorable.

Conclusion: Comprehensive assessment of effects and AEs is of imminent importance to create standardized management algorithms in order to improve DBS treatment in young patients. 


\section{Quantification of instability of tone production in embouchure dystonia}

\author{
Lee A. ${ }^{1,2}$, Furuya S. ${ }^{2,3}$, Morise M. ${ }^{4}$, Altenmüller E. ${ }^{2}$
}

${ }^{1}$ Klinikum rechts der Isar, Technische Universität München, Department of Neurology, München, Germany, ${ }^{2}$ University of Music, Drama and Media Hannover, Institute for Music Physiology and Musicians' Medicine, Hannover, Germany, ${ }^{3}$ Sophia University, Department of Information and Communication Sciences, Tokyo, Japan, ${ }^{4}$ University of Yamanashi, Interdisciplinary Graduate School of Medicine and Engineering, Kofu, Japan

Objective: Musician's dystonia is a task-specific loss of voluntary control of the motor system such as fingers or the embouchure. In contrast to pianists' dystonia which can be objectively assessed based on movement kinematics and muscular activities, no quantitative measure has been established for embouchure dystonia.

Methods: We focused on acoustic signals, and investigated, whether the fluctuation of the time-varying fundamental frequency of a note can provide an objective and reliable measure of embouchure dystonia. Results: A comparison between patients with embouchure dystonia and healthy controls found a significantly higher variability of the fundamental frequency for the patients.

Conclusion: The present findings propose a new quantification method for embouchure dystonia.

\section{Fixed dystonia of the left hand in a violinist, a rare functional disorder}

\author{
Lee A. ${ }^{1,2}$, Jahnke A. ${ }^{3}$, Altenmüller E. ${ }^{2}$ \\ ${ }^{1}$ Klinikum rechts der Isar, Technische Universität München, \\ Department of Neurology, München, Germany, ${ }^{2}$ University of Music, \\ Drama and Media Hannover, Institute for Music Physiology \\ and Musicians' Medicine, Hannover, Germany, ${ }^{3}$ St. Joseph's \\ Hospital, Ennis, Ireland
}

Background: Fixed dystonia leads to an immobile abnormal posturing of the affected limb. It is an ongoing debate whether this condition is psychogenic in origin.

Case report: We present a 21 year old violinist with fixed dystonia after an acute overuse injury with a transient cyanosis but no signs for psychological trauma. After Incobotulinumtoxin-injection symptoms subsided within $8 \mathrm{~h}$.

Discussion: Our case corroborates the notion that fixed dystonias after minor injuries are functional disorders. It underlines the necessity of a biopsychosocial approach to functional disorders, considering the possibility of an overlay between organic and non-organic disorders.

\section{Coherence of coactivation and acceleration in task- specific primary bowing tremor}

Lee A. ${ }^{1,2}$, Tominaga K. ${ }^{3}$, Furuya S. ${ }^{2,4}$, Miyasaki F. ${ }^{3}$, Altenmüller E. ${ }^{2}$

${ }^{I}$ Klinikum rechts der Isar, Technische Universität München, Department of Neurology, München, Germany, ${ }^{2}$ University of Music, Drama and Media Hannover, Institute for Music Physiology and Musicians' Medicine, Hannover, Germany, ${ }^{3}$ Osaka University, Department of Engineering Science, Osaka, Japan, ${ }^{4}$ Sophia University, Department of Information and Communication Sciences, Tokyo, Japan
Background: Primary bowing-tremor is a task-specific tremor that may occur in the right arm of violinists. We recently showed that a coactivation of wrist antagonist muscles, however, a direct relationship could not be shown between either coactivation and tremor or between EMG-activity and tremor.

Methods: Coherence between coactivation and tremor and EMGactivity and tremor was assessed.

Results: We found coherence between coactivation of wrist antagonist muscles and tremor as well as between EMG-activation and tremor in patients at a frequency-range of 3-8 Hz. No coherence was found in healthy controls.

Conclusion: Our findings show a direct relationship between coactivation and tremor. The direct relationship of antagonist coactivation and tremor may indicate that task-specific tremors are a manifestation of task-specific dystonia, where coactivation is a main feature. The coherence between EMG-activity and tremor corroborates our previous notion that PBT emerges mainly due to central neurogenic contributions via motor-unit-synchronization.

\section{Homozygous mutations in SACS, ATCAY, and MCOLN1 in three Pakistani families with dystonic features}

Lohmann K. ${ }^{1}$, Manzoor H. ${ }^{1,2}$, Bäumer T. ${ }^{1}$, Hinrichs F. ${ }^{1}$, Hussain H.M.J. ${ }^{3}$, Wajid M. ${ }^{4}$, Naz S. ${ }^{2}$, Brüggemann N. ${ }^{1,5}$, Münchau A. ${ }^{1,6}$

${ }^{1}$ University of Luebeck, Institute of Neurogenetics, Lübeck, Germany,

${ }^{2}$ University of the Punjab, School of Biological Sciences, Lahore, Pakistan, ${ }^{3}$ University of the Punjab, Department of Microbiology and Molecular Genetics, Lahore, Pakistan, ${ }^{4}$ University of Education, Lahore, Pakistan, ${ }^{5}$ University of Luebeck, Department of Neurology, Lübeck, Germany, ${ }^{6}$ University of Luebeck, Department of Paediatric and Adult Movement Disorders and Neuropsychiatry, Lübeck, Germany

Introduction: A number of genetic causes of autosomal dominant dystonias have been identified, however genes for recessive forms remain largely unknown. Recessive causes can best be elucidated in consanguineous families using next generation sequencing.

Methods: We collected five Pakistani consanguineous families with dystonic features in multiple affected individuals. Patients were videotaped and clinically evaluated by neurologists. We performed exome sequencing in 4-5 samples per family. Variants were filtered based on homozygosity in patients, predicted protein-sequence change, and frequency in public databases. Segregation of candidate variants was tested in all available family members by Sanger sequencing.

Results and discussion: In Family RDHM-01, the disease was characterized by ataxia of gait, bradykinesia including hypomimia, mild dystonic postures of upper limbs, supranuclear gaze palsy, and spasticity with onset at the age of 1.5 year. In all four patients, we identified a novel, homozygous 1-basepair duplication (c.9119dup; p.Asn3040fs) in the SACS encoding sacsin, mutations in which caused spastic ataxia of the Charlevoix-Saguenay type. In Family RDHR-04, a homozygous 7-basepair deletion (c.599_605del; p.Pro200fs) was found in all five patients in ATCAY encoding caytaxin, mutations in which cause Cayman cerebellar ataxia. Our patients had severe gait ataxia, mild bibrachial dystonia, strabism, apraxia, and a suspicion of cognitive impairment with onset at the age of about 4 months. Finally, in Family RDHM-03, a homozygous missense mutation (c.551T >C; p.Ile184Thr) was found in MCOLN1 encoding mucolipin 1. Both patients in this family had adolescence-onset generalized 
dystonia. Of note, mutations in MCOLN1 cause mucolipidosis IV, a neurodegenerative lysosomal storage disorder. None of these mutations was found in 200 ethnically matched controls. In conclusion, exome sequencing is a powerful tool to detect disease-causing variants particularly in genes that would not be picked for diagnostic testing by Sanger sequencing.

\section{PEDiDBS: On the introduction of the international pediatric DBS registry}

\author{
Marks W. ${ }^{1,2}$, Sanger T. ${ }^{3}$, Bailey L. ${ }^{1,4}$, Koy A. ${ }^{5}$, Lin J.-P. ${ }^{6}$, Mink J. ${ }^{7}$, \\ Timmermann L. 5 \\ ${ }^{1}$ Cook Children's Medical Center, Neurosciences, Fort Worth, TX, \\ USA, ${ }^{2}$ University of North Texas Health Sciences Center, Pediatrics, \\ Fort Worth, TX, USA, ${ }^{3}$ University of Southern California, Los \\ Angeles, CA, USA, ${ }^{4}$ University of North Texas Health Sciences \\ Center, Fort Worth, TX, USA, ${ }^{5}$ University of Cologne, Cologne, \\ Germany, ${ }^{6}$ Evelina Children's Hospital, London, United Kingdom, \\ ${ }^{7}$ University of Rochester, Rochester, NY, USA
}

\begin{abstract}
Although deep brain stimulation has been utilized for adult patients since 1997, DBS has only been available for the treatment of pediatric patients with dystonia since 2003. In the United States, there is an FDA HDE imposed lower age limit of 7 years. Although a few centers have developed significant experience with pediatric patients, most children worldwide are implanted in adult centers or pediatric centers with small surgical volumes. Additionally, there is a low incidence of disorders for which DBS may be applicable in childhood. There are many questions about the role of DBS in children that can only be answered by collective shared experience. An international registry has been developed to capture experience from centers large and small regarding pediatric patients undergoing DBS implantation. The registry is open to any center implanting a child aged 21 years or younger with DBS. There is no restriction with regard to the underlying disorder, implant site, surgical technique. Participation is voluntary. A limited data set is utilized and data is stored securely in accordance with privacy laws. Sites are identified only by number and retain access to their own data. A data oversight committee is responsible for the management of access to collective data for research purposes. Information and request for participation is available at http://www.pedidbs.org.
\end{abstract}

\section{Deep brain stimulation in pediatrics: the dystonia experience at Cook Children's Medical Center}

\author{
Marks W. ${ }^{1,2}$, Bailey L. ${ }^{1,2}$, Reed M. ${ }^{1}$, Acosta F. ${ }^{1}$, Honeycutt J. ${ }^{1}$ \\ ${ }^{1}$ Cook Children's Medical Center, Fort Worth, TX, USA, ${ }^{2}$ University \\ of North Texas Health Sciences Center, Fort Worth, TX, USA
}

Introduction: Deep brain stimulation received United States approval for dystonia in 2003. Since 2007 we have offered Deep Brain Stimulator (DBS) placement in pediatric movement disorder patients at Cook Children's Medical Center. We have modified our surgical approach to help increase efficacy and minimize morbidity. Methods: A retrospective review was performed on our first 100 DBS patients. All patients enrolled for DBS surgery at Cook Children's Medical Center undergo an IRB approved consent process in accordance with the USFDA Humanitarian Device Exemption status of this device for dystonia.
Results: One hundred patients underwent primary implants. Among our dystonia patients, the first 83 patients were done awake with microelectrode recordings (MER). Our last $17 \mathrm{GPi}$ lead placements were done under general anesthesia using the Clearpoint MRI guided system. TMean aCP patients with a slight male predominance in both groups. Primary dystonias accounted for $30 \%$ of MER and $56 \%$ of CP implants. Surgical complications among MER patients included 11 infections in 8 patients, strokes or hemorrhages in 7 patients. Eleven MER patients required lead relocation for inadequate efficacy. In the $\mathrm{CP}$ group, there has been one epidural hemorrhage during frame placement and one infection (5.0\%). Postsurgical hardware complications include 8 extension wire and 4 lead fractures, all in patients with dystonia.

Conclusion: Over the last 9 years, we have modified our surgical approach to minimize complications (i.e., more lateral trajectory to decrease strokes/hemorrhage, two-stage placement to decrease infection, and asleep surgery to increase comfort). DBS remains an important tool in the management of carefully selected patients with medically refractory movement disorders. Although complications remain problematic in the pediatric population, they can be reduced with experience and improvements in surgical techniques.

\section{Botulinum toxin treatment of freezing of gait in Parkinson's disease patients as reflected in functional magnetic resonance imaging of leg movement}

Vastik M. ${ }^{1}$, Hlustik P. ${ }^{1}$, Otruba P. ${ }^{1}$, Mensikova K. ${ }^{1}$, Hok P. ${ }^{1}$, Tudos Z. $^{2}$, Kanovsky P. ${ }^{1}$

${ }^{1}$ Palacky University Medical School, Department of Neurology, Olomouc, Czech Republic, ${ }^{2}$ Palacky University Medical School, Department of Radiology, Olomouc, Czech Republic

Background: Freezing of gait (FOG) is a common disabling symptom in Parkinson's disease (PD). The mechanism of FOG in not clearly understood, nevertheless, the dystonia of lower limb muscles was discussed. We investigated the clinical effect and changes of the activity of the sensorimotor system using functional MRI (fMRI) examinations before and after application of botulinum toxin in Parkinson's disease patients with FOG.

Methods: 20 patients with PD, 10 with FOG and 10 without FOG were examined. PD patients with FOG were treated with intramuscular application of botulinum toxin type A into the tensor fasciae latae muscle bilaterally. The clinical effect of treatment was assessed using FOG questionnaire, "Time up and go" test, UPDRS, Hoehn and Yahr staging and GCI scale. Activation of the sensorimotor system was studied using BOLD fMRI of the whole brain during repetitive abduction-adduction of each leg interleaved with rest. The clinical (in the FOG group) and imaging (in both groups) examination was repeated after a four-week interval.

Results: In the FOG group, the FOG questionnaire has shown a decline of scores after application of botulinum toxin that suggests possible effect of botulinum toxin on freezing of gait. In fMRI results, both groups manifested reduction of the sensorimotor network activated with leg movement, however, the FOG group also showed increased activation in cerebellar vermis and nuclei, in dorsal pons and in medulla after treatment.

Conclusion: Alleviation of the FOG in PD patients by botulinum toxin seems to be reflected in the functional participation of the cerebellum and its projections as seen by fMRI.

Supported by Institutional Support of Research organization MZ CR RVO-FNOL 2016. 
The effects of internal globus pallidus deep brain stimulation on dystonia - preliminary results

\author{
Merrouche B. ${ }^{1}$, Guenane L. ${ }^{1}$, Abdennebi B. ${ }^{1}$ \\ ${ }^{I}$ Faculty of Medicine, Hospital Salim Zemirli, Department \\ of Neurosurgery, Algiers, Algeria
}

Introduction: Dystonia is a syndrome characterized by involuntary sustained muscle contractions that result in twisting, repetitive movement and abnormal posture. Dystonia is typically classified by age of onset, origine, and affected body region. When the cause is not defined or unknown, the dystonia is referred to as idiopathic or primary dystonia. Primary dystonia can be familial.

Materials and methods: 27 patients (15 males and 12 females) underwent this surgical technique. Ranging in AGE from 07 to 57 years, generalized dystonia (19 patients) and localized (08 patients), Electrodes were bilaterally implanted under stereotactic guidance and connected to neurostimulateurs. Efficacy was evaluated by comparing scores on the clinical and functional Burke-MarsdenFahn dystonia rating Scales (BMFDRS) before and after implantation (3 and 6 months and 1 year postoperatively). The operation was performed under standard general anesthesia.

Results: After 3 months the improvement of the clinical score was $40 \%$, the functional score was improved by $22 \%$. After that, at 6 months it was respectively: clinical: $56 \%$ and functional: $41 \%$; Finally at 1 year: the improvement concerned the clinical score: $80 \%$ and the functional score: $85 \%$.

Conclusion: Bilateral chronic electrical stimulation can be proposed as first line treatment for generalized dystonia. It is conservative, adaptable, reversible and well tolerated by the pediatric population. It must be applied as soon as possible, especially in primary dystonia.

Tolerance is excellent and the complication rate remains low.

\section{Task-specific dystonia in Brazilian musicians}

\author{
Moura R.D.C. ${ }^{1}$, Aguiar P.M. ${ }^{2}$, Bortz G. ${ }^{3}$, Ferraz H.B. ${ }^{1}$ \\ ${ }^{1}$ Federal University of São Paulo, Neurology, São Paulo, Brazil, \\ ${ }^{2}$ Hospital Albert Einstein, Brain Institute, São Paulo, Brazil, ${ }^{3}$ State \\ University of São Paulo, São Paulo, Brazil
}

Background: Musician's dystonia is a task-specific focal dystonia that can occur in any part of the body, most commonly on the face, neck muscles and upper limbs. Its frequency among the Brazilian population is unknown. This study aimed to assess the complaints of musicians with performance problems attributed to motor difficulties and to assess the frequency and clinical profile of musician's dystonia in Brazil.

Methods: To identify individuals with dystonia, we visited orchestras and music schools in 19 Brazilian cities between March 2013 and March 2015, providing information about task-specific dystonia (TSD). Musicians were invited to answer a questionnaire, and those with possible neurological dysfunction were filmed while performing. In total, 2232 questionnaires were completed, with 72 subjects recorded on video in specific tasks and also were submitted the assessment of anxiety states and the severity of dystonia.

Results: In total, 49 individuals were diagnosed with TSD (mean age 36.4 ( \pm ) 12.2 years; $92 \%$ male). The instruments most associated with TSD was the guitar acoustic $(36.75 \%)$ and the brass instruments $(30.6 \%)$.

Conclusion: Our results suggest that the frequency of focal dystonia among musicians in Brazil is $2.2 \%$, it can initially appear around 30 years old and is mostly associated with males, professional musicians who play classical concert music and in people who perform other activities using their upper limbs. Musculoskeletal injuries appear prior to the onset of dystonia. TSD is a condition that deserves further attention from musicians, teachers and health professionals.

\section{Changing treatment between Ona-botulinumtoxin-A and Abo-botulinumtoxin-A in cervical dystonia, hemifacial spasm and blefarospasm. A cross-over study}

\author{
Nazarian N. ${ }^{1}$, Danielsen E. ${ }^{1}$ \\ ${ }^{1}$ Aarhus University Hospital, Dept. of Neurology, Aarhus, Denmark
}

Introduction: No guidelines are available for changing treatment between the different botulinum neurotoxins (BoNTs).The aim of this study was to find the effective conversion ratio between Ona-botulinumtoxin-A (Botox ${ }^{\circledR}$ )/Abo-botulinumtoxin-A (Dysport ${ }^{\circledR}$ ) therapy. In addition, the effect of using Ona-botulinumtoxin-A and Abo-botulinumtoxin-A on patients with dystonia, who have been followed up in the range of 1991-2014, is reported.

Methods: The doses of Ona-botulinumtoxin-A were collected for minimum a year when stable optimal treatment doses were achieved. Each patient was then changed to treatment with Abo-botulinumtoxin-A. The effects were estimated by the visual analogue scale (VAS). The average conversion ratio of Ona-botulinumtoxin-A/Abobotulinumtoxin-A was estimated when stable optimal treatment doses were found.

Results: 55 patients were included in this study. 4 patients had blepharospasms and treatment time was $13.25 \pm 2.21$ years. We samples 126 injection series. Doses were $27.075 \pm 10.63$ MU-Onabotulinumtoxin-A and 75.71 \pm 36.04 MU-Abo-botulinumtoxin-A. 3 patients had hemifacial spasms- treatment time was $13 \pm 3$ years and we sampled 125 injection series. Doses were $14.02 \pm 8.06 \mathrm{MU}-$ Onabotulinumtoxin-A and $63.75 \pm 53.657$ MU-Abo-botulinumtoxin-A. 48 patients had cervical dystonia- treatment time was $15.75 \pm 4.58$ years and we sampled 2546 injection series. Doses were $126 \pm 38.77 \mathrm{MU}$ - Ona-botulinumtoxin-A and $381.6 \pm 151.1$ Abo-botulinumtoxin-A.The effect range is $20-100 \%$ by both BoNTs. The conversion ratio for blepharospasm was $1: 2.94$. For hemifacial spasm 1: 3.03 , and for cervical dystonia the ratio was $1: 2.56$.

Discussion: By considering the effects, the conversion ratios and the long follow-up period, this study shows that it is safe and satisfying for changing treatment from Ona-botulinumtoxin-A to Abo-botulinumtoxin-A at the found conversion ratios.

\section{Subtle sensory abnormalities in patients with segmental and cervical dystonia detected by quantitative sensory testing}

Paracka L. ${ }^{1}$, Wegner F. ${ }^{1}$, Blahak C. ${ }^{2}$, Abdallat M. ${ }^{3}$, Karst M. ${ }^{4}$, Dressler D. ${ }^{1}$, Krauss J.K. ${ }^{3}$

${ }^{1}$ Hannover Medical School, Department of Neurology, Hannover, Germany, ${ }^{2}$ University of Heidelberg, Faculty of Medicine Mannheim, Department of Neurology, Heidelberg, Germany, ${ }^{3}$ Hannover Medical School, Department of Neurosurgery, Hannover, Germany,

${ }^{4}$ Hannover Medical School, Department of Anesthesiology, Hannover, Germany 
Sensory abnormalities are increasingly being recognized as an accompanying symptom in patients with isolated inherited or idiopathic dystonia. The aim of this study was to investigate whether sensory abnormalities would be related to age and to the distribution of motor symptoms in patients with primary dystonia. For this purpose we recruited 20 dystonic patients, from which 8 had generalized dystonia, 7 cervical dystonia and 5 segmental dystonia with hand involvement and compared them with age matched controls. We used Quantitative Sensory Testing (QST) on the back of the hand in all patients and on the shoulder in patients with cervical dystonia. The patients with involvement of the hand in dystonia were divided into two groups: younger than 40 years of age (6 patients) and older than 40 (7 patients). All patients with cervical dystonia were older than 40 . The QST values were compared to 19 age matched controls. Our results show that younger patients with hand dystonia have both impaired cold pain threshold (CPT) and hot pain threshold (HPT) on the side more affected by dystonia, and also impaired mechanical pain sensitivity (MPS) on both sides. Patients older than 40 have a diminished cold detection threshold (CDT) and thermal sensory limen (TSL) on the side more affected by the disease and diminished MPS of the hand on both sides. Patients with cervical dystonia have an increased hot detection threshold (HDT), CDT, TSL and MPS on the back of the hand. In the group with cervical dystonia, the shoulder QST revealed impaired CDT and MPS. QST clearly shows several subtle sensory abnormalities in patients with inherited or idiopathic dystonia. Whether or not altered sensory function in dystonic patients correlates with the degree of dystonia needs further clarification.

Key words: Dystonia, QST, sensory abnormalities.

The first and second authors contributed equally.

\section{The Technical Ability and Performing Scale (TAPS): a proposal of a new functional rating scale for Musicians Focal Dystonia (MFD)}

\section{$\underline{\text { Ramella M. }}{ }^{1}$, Giacobbi G. ${ }^{1}$, Castagna A. ${ }^{1}$, Converti R.M. ${ }^{1}$ \\ ${ }^{I}$ Don Gnocchi Foundation IRCCS Santa Maria Nascente, Milano, Italy}

Objective: The aim of this study is to present a new functional rating scale for MFD.

Background: The available rating scales for MFD suffer from lack of sensitivity to verify the clinical changes, especially during rehabilitation, and are not digit-specific.

TAPS is a self-evaluation scale made up of seven visual analogical scales. Six items investigate the motor disorder extent while the musician is playing different technical passages (TP) while the seventh evaluate the last week global performance (PS). The Technical Ability (TA) is the six TP median value. The six TP are: the chromatic scale at three increasing speed (V1, V2, V3), the C-major scale ascending/descending (CS), a piece and a TP chosen by musician. For drummers instead of $\mathrm{V} 1, \mathrm{~V} 2, \mathrm{~V} 3$ and $\mathrm{CS}$ we asked to perform two basic TP and one of them at three increasing speed. All the items had to be played for $45 \mathrm{~s}$ at specific speed. The musician had to assign the score just at the performance end. If they were unable to complete the task we assign them a zero.

Methods: We evaluated 38 patients with the TAPS, the Arm Dystonia Disability Scale (ADDS). All musical instruments and type of MFD were included. The data were statistically analyzed with the Spearmen rank order correlation test and Cronbach's.

Results: Cronbach's among the six TP was 0.79 . TA was positively correlated with all TP $(\mathrm{p}<0.05)$ and negatively with ADDS $(\mathrm{r}=-0.36, \mathrm{p}<0,05)$, the age $(\mathrm{r}=-0.3, \mathrm{p}<0.05)$ and the onset age $(\mathrm{r}=-0.40, \mathrm{p}<0.05)$. PS is significantly correlated only with ADDS. TA doesn't correlate with PS.

Conclusions: The data had showed that TAPS is a tailored useful tool to evaluate the FD subjective perception and the quality of performance.

\section{A new integrated rehabilitation protocol to treat musician focal dystonia (MFD)}

\author{
$\underline{\text { Ramella M. }}{ }^{1}$, Bologna C. ${ }^{1}$, Giacobbi G. ${ }^{1}$, Castagna A. ${ }^{1}$, \\ Converti R.M. ${ }^{1}$ \\ ${ }^{1}$ Don Gnocchi Foundation IRCCS Santa Maria Nascente, Milano, \\ Italy
}

Objective: The aim of this study is to assess the effectiveness of an integrated rehabilitation protocol, based on SaM (Sense and Mind) Method's and Mirror Therapy's principles on MFD.

Background: MFD is a movement disorder affecting 1 in $200-500$ instrumental musicians and can lead to the end of the musical carrier. A variety of treatments have been proposed such as oral medications, retraining, biofeedback, constraint-induced therapy, splinting, botulinum toxin. The Mirror Therapy, such as Laterally Construction, Motor Imagery and Mirror Box, has been used for the treatment of phantom limb pain, complex regional pain syndrome and stroke rehabilitation. SaM Method is a spatial rehabilitation approach which has the goal to improve, through specific motor exercises, perception and action; it has been used to treat TBI, stroke rehabilitation.

Methods: Fourteen musicians affected by FD, referred to the "Sol Diesis Service" of the Don Gnocchi Foundation, were included in the study. All musical instruments and type of FD were included. The patients followed a 10 weeks protocol. All musicians had to evaluate themselves before and after the treatment by using the Technical Ability and Performing Scale (TAPS). TAPS is a functional scale made up of visual analogical scale that investigates the motor disorder extent while musicians are playing six different musical items and the global performance (PS). The Technical Ability (TA) is the six technical items' median. The data were statistically analyzed with the Wilcoxon rank test.

Results: The data analysis showed statistically significant changes for TA $(\mathrm{p}<0.005)$ for TA and for PS $(\mathrm{p}<0.001)$.

Conclusions: Further investigation is necessary, but the integrated rehabilitation protocol appears to be a useful complementary treatment for musician affected by FD.

\section{A combined behavioural and mindfulness programme for people living with Dystonia: results of a feasibility study}

Sandhu H. ${ }^{1}$, Bernstein C. ${ }^{2}$, Davies G. ${ }^{3}$, Tang N.K.Y. ${ }^{2}$, Belhag M. ${ }^{4}$, Tingle A. ${ }^{5}$, Field M. ${ }^{5}$, Foss J. ${ }^{2}$, Lindahl A. ${ }^{4}$, Underwood M. ${ }^{2}$, Ellard D. ${ }^{2}$

${ }^{1}$ University of Warwick, Clinical Trials Unit, Coventry, United Kingdom, ${ }^{2}$ University of Warwick, Coventry, United Kingdom, ${ }^{3}$ University of Southampton, Southampton, United Kingdom, ${ }^{4}$ University Hospitals Coventry and Warwickshire NHS Trust, Coventry, United Kingdom, ${ }^{5}$ The Dystonia Society (West Midlands Group), Solihull, United Kingdom

Introduction: Dystonia (a neurological condition) refers to a range of movement disorders typically characterised by tremor and movements 
affecting one or more sites of the body and/or abnormal postures from sustained muscle contractions. Quality of life (QoL) can be diminished leading to higher risks of depression and anxiety. There is a lack of evidence for psychological and behavioural interventions, improving QoL in Dystonia. Mindfulness is shown to improve patient health outcomes for those with chronic conditions. The aim of this study was to test the feasibility of an intervention integrating psychological, behavioural and mindfulness principles for those living with dystonia. Methods: A total of 12 participants (from a NHS Botulinum Toxin Clinic) consented to attend a three-day group residential programme led by a psychologist and a mindfulness expert. A detailed process evaluation consisting of pre and post participant interviews and programme observation was completed. Quantitative data for health and wellbeing, mood and anxiety were collected at baseline, 1- and 3-month using validated questionnaires.

Results and discussion: Nine participants completed the whole programme. Analysis of interview data (using NVIVO) showed that overall participants found the programme useful and it was very well liked. Learning about their condition was insightful, and they felt they had new strategies to help self-manage their dystonia. The mindfulness was received positively with all participating in the practical sessions. The residential and group format was received positively. There was a positive trend indicated in mean scores for wellbeing, anxiety, depression and global dystonia.

In conclusion, we have successfully delivered a three-day residential programme to help those living with dystonia combining psychological, behavioural and mindfulness principles. Feedback was useful and will be considered in the next stage of a randomised controlled trial to test the efficacy and cost effectiveness of the programme against best usual care.

\section{Deafness-dystonia syndrome}

Schinwelski M. ${ }^{1,2}$, Dulski J. ${ }^{1}$, Mandat T. ${ }^{3}$, Pienczk-Ręcławowicz K. ${ }^{4}$, Sławek J. ${ }^{1,2}$

${ }^{1}$ St Adalbert Hospital Copernicus PL, Gdansk, Poland, Neurology Department, Gdansk, Poland, ${ }^{2}$ Medical University of Gdansk,

Gdansk, Poland, Neurological and Psychiatric Nursing Department, Gdansk, Poland, ${ }^{3}$ Department of Neurosurgery, Maria SktodowskaCurie Institute of Oncology, Warszawa, Poland; Department of Neurosurgery, Institute of Psychiatry and Neurology, Warszawa, Poland, Warszawa, Poland, ${ }^{4}$ Department of Developmental Neurology, Medical University of Gdansk, Gdansk, Poland

In this report we present a patient with deafness-dystonia syndrome of unknown cause, refractory to medical treatment, who has been successfully treated with deep brain stimulation of the internal globus pallidus (GPi-DBS) and followed-up for 4 years. To our knowledge, only two cases of this syndrome treated with deep brain stimulation have been reported till now. The Burke-Marsden-Fahn's Dystonia Rating Scale improved from 75 points before surgery to 10 and 15 points, at 3 months and 4 years, respectively, after the surgery.

\section{Usefulness of segmented leads in anatomical variants of the brain}

\author{
Slotty P. ${ }^{1}$, Vesper J. ${ }^{1}$ \\ ${ }^{1}$ University Düsseldorf, Functional Neurosurgery and Stereotaxy, \\ Düsseldorf, Germany
}

Introduction: DBS is well known to be effective for suppressing various movement disorders. Both primary and secondary dystonia are improved by DBS. Potential limits due to side effects, may occur especially in the proximity of internal capsule. Recently segmented leads where introduced to reduce side effects and more efficiently stimulate in the desired direction within the target area. We hereby report on the first case of dystonia which was treated with those directional segmented leads.

Methods: A 31-year-old female was suffering from generalized dystonia for more than 20 years. Potentially symptoms were caused by an ataxia with isolated Vitamin E deficiency syndrome. She underwent DBS surgery using direct targeting of the left GPI. Standard coordinates did not match the individual anatomy of the patient with distortion of the basal ganglia left and a hyperplastic posterior horn of the lateral ventricle. Patient was completely wheelchair bounded and suffered from severe dysarthria.

Results: After calculating standard coordinates (3.5 ant, 22 lateral and 4.0 below MCP), trajectory was adapted visually to the lateral border of the optic tract. The posterior communicating artery which atypically went over the optical tract was further limiting the approach. With 3 leads for MER surgery was conducted and electrode (Vercise DBS, BSCI) was placed. Conventional stimulation caused a fast additional dysarthria and painful stimulation side effects. Directional stimulation on the left site provided one segment anteriorly, 1 posterolateral and one posteromedial. The segmented contacts were programmed with the least intensity. After 3 months the patient is able to walk without caregivers help. Dysarthria was also significantly improved.

Conclusion: Directional segmented leads offer new perspectives for DBS while reducing side effects and energy consumption. Further investigations are warranted to evaluate their impact for improving outcome and in general facilitating surgery to increase the acceptance of this promising procedure.

\section{Treatment of cervical dystonia (CD) with BoNT: volume measurements of $M$. obliquus capitis inferior (OCI) in 56 patients with and 23 without torticaput}

\author{
Stenner A. ${ }^{1}$, Reichel G. ${ }^{1}$, Jahn A. ${ }^{2}$ \\ ${ }^{1}$ Paracelsus Clinic, Movement disorders, Zwickau, Germany, \\ ${ }^{2}$ Radiologic Praxis, Zwickau, Germany
}

The classification of CD in 4 categories was replaced in 2009 (Reichel et al.) with a more subtle classification into 11 types ( 4 collis and caput types and 3 shifts). Although a clear correlation between the muscles involved and the clinical findings exists, this is not clear for the OCI muscle, which often is increased in diameter in torticaput. To investigate whether the OCI is dystonic or just being passively squeezed by the rotation of the lower atlanto-axial joint by other dystonic muscles, we measured left and right OCI volume differences by $\mathrm{CT}$ in 56 torticaput and 23 nondystonic patients (statistics: MATLAB by Max Doberenz). If the head is deflected in nondystonic patients to one side, the ipsilateral OCI is compressed and is higher in volume that on the other side $(0.2-0.5 \mathrm{ccm})$. The mean difference between both OCI volumes in 56 torticaput patients was $1.50 \pm 0.88 \mathrm{ccm}$. If one subtracts the standard deviation value from the nondystonic patients and allows for the standard deviation of the measurements in OCI patients, and the volume difference is still positive, the muscle is contracted, i.e., dystonic. This was the case in 25 of 38 patients. Deflection of the head gives a shortening and thickening of the ipsilateral OCI in nondystonic patients. The volume of the muscle does not change significantly. In patients with torticaput, the volume difference between the OCI is increased, obviously a sign of a volume increase in the dystonic OCI. In detail: if the OCI 
ipsilateral to the side to which the head is deflected is $1.013 \mathrm{ccm}$ or larger than that on the other side, it is dystonically contracted.

The measurement of OCI volume in torticaput patients can determine whether it is dystonically contracted. This was true in $62 \%$ of our sample of patients with torticaput.

\section{Assessment of patient preference and clinical need for flexible injection intervals in incobotulinumtoxin $A$ treatment of cervical dystonia and blepharospasm}

\author{
Truong D. ${ }^{1}$, Sethi K.D. ${ }^{2}$, Fezza J. ${ }^{3}$, Evidente V.G.H. ${ }^{4}$ \\ ${ }^{1}$ The Parkinson's and Movement Disorder Institute, Fountain Valley, \\ CA, USA, ${ }^{2}$ Georgia Regents University, Augusta, GA, USA, ${ }^{3}$ Center \\ for Sight, Sarasota, FL, USA, ${ }^{4}$ Movement Disorders Center \\ of Arizona, Scottsdale, AZ, USA
}

Introduction: Botulinum toxin type A (BoNT-A) injections are indicated for the treatment of cervical dystonia (CD) and blepharospasm. Minimum injection intervals of 12 weeks are generally recommended ( $\geq 10$ weeks in Europe for incobotulinumtoxinA), despite common symptom recurrence between treatments. We investigated the medical need for more flexibility in injection intervals.

Methods: We conducted surveys with patients who had completed $\geq 2$ BoNT-A treatment cycles. Patients with CD were interviewed 7-12 weeks after their last BoNT-A injection; patients with blepharospasm were interviewed immediately before re-injection. Survey data were compared with data from clinical trials that allowed incobotulinumtoxinA $\left(\mathrm{Xeomin}^{\circledR}{ }^{\circledR}\right.$ ) treatments at flexible intervals ( $\geq 6$ weeks) based on patient request and investigator-assessed clinical need.

Results and discussion: We surveyed 136 patients with CD and 141 with blepharospasm [mean (standard deviation; SD) disease duration of 92.9 (86.2) and 138.7 (111.0) months, respectively]. The mean (SD) treatment interval patients usually received was 14 (3.7) weeks for patients with $\mathrm{CD}$ and 12.5 (3.3) weeks for patients with blepharospasm. However, the majority of patients indicated a preference for BoNT-A-treatment intervals $\leq 12$ weeks $(\mathrm{CD}, 78.4 \%$; blepharospasm, $69.5 \%$ ), and many patients would prefer intervals $\leq 10$ weeks (CD, 46.3\%; blepharospasm, 43.4\%). In clinical trials allowing flexible intervals (based on patient request and investigatorassessed clinical need), $44.9 \%$ of CD treatments and $44.9 \%$ of blepharospasm treatments were administered after intervals $<12$ weeks. Furthermore, $29.5 \%$ of CD treatments and $26.5 \%$ of blepharospasm treatments were required after intervals $<10$ weeks. The overall incidence of adverse events was comparable between injection intervals $<12$ weeks and $\geq 12$ weeks in both indications.

In conclusion, patient survey and clinical trial data indicate that many patients with $\mathrm{CD}$ or blepharospasm have a preference and a medical need for BoNT-A-treatment intervals shorter than the 12-week standard interval, and that treatment interval flexibility does not compromise incobotulinumtoxinA tolerability.

\section{Effect of Deep brain stimulation on sleep and pain in dystonia patients}

\author{
Vuletic V. ${ }^{1,2}$, Chudy D. ${ }^{3}$, Almahariq F. ${ }^{3}$ \\ ${ }^{1}$ University Hospital Dubrava, Department of Neurology, Zagreb, \\ Croatia, ${ }^{2}$ Faculty of Medicine, University of Rijeka, Rijeka, Croatia, \\ ${ }^{3}$ University Hospital Dubrava, Department of Neurosurgery, Zagreb, \\ Croatia
}

Background: There is rather high frequency of sleep problems and pain in dystonia patients. They are often neglected and untreated but affect significantly quality of life. Our aim was to see the effect of Deep brain stimulation (DBS) on sleep and pain in dystonia patients.

Methods: We conducted investigation with anamnesis and treatments' data, Burke-Fahn-Marsden Dystonia Rating Scale (BFMDRS), Pittsburgh Sleep Quality Index (PSQI), Visual Analogue Scale, McGill questionnaire and Hospital Anxiety and Depression Scale (HADS). The study involved 10 patients with generalized dystonia. We did the basal testing before DBS and another investigation after 6 months. In addition, we try to see factors that were connected with pain and sleep problems.

Results: Six months after DBS we observed significant improvement measuring by BFMDRS score [from 55 to 15.2 $(72.3 \%, \mathrm{p}<0.05)]$ and decrease in frequency concerning sleep problems (from 90 to $10 \%$ of patients) and pain (from 90 to 20\%) $(\mathrm{p}<0.05)$. We found that both symptoms were associated with depression.

Conclusions: DBS, beside motor improvement, helps in relieving of the pain and sleep problems in dystonia patients.

\section{Multimodal physiotherapy as an add-on treatment to botulinum neurotoxin therapy in patients with cervical dystonia}

\author{
Werner C. ${ }^{1}$, Derlien S. ${ }^{1}$, Witte O.W. ${ }^{2}$, Günther A. ${ }^{2}$, Smolenski U.C. ${ }^{1}$ \\ ${ }^{1}$ Universitätsklinikum Jena, Institut für Physiotherapie, Jena, \\ Germany, ${ }^{2}$ Universitätsklinik Jena, Hans-Berger-Klinik für \\ Neurologie, Jena, Germany
}

Introduction: Treatment of choice for cervical dystonia (CD) comprises a local injection of botulinum neurotoxin type A (BoNT-A). Whether additional physiotherapy can achieve better results is not clearly documented in the literature. The present study aims to show possible effects of physiotherapy as an add-on treatment to therapy with botulinum neurotoxin.

Methods: 18 Patients with CD were enrolled in a prospective singlecenter interventional study. Study duration comprised 6 months and was divided into two phases with each phase lasting for 3 months. First phase contained the usual BoNT-A application. Second phase comprised a multimodal physiotherapy program consisting of two 45 min sessions per week in addition to the BoNT-A treatment. Primary outcome measures were quality of life using the SF-36 score and the range of motion (ROM) determined by an ultrasonic based measurement system (ZEBRIS). Secondary outcome measures included the Toronto Western Spasmodic Torticollis Rating Scale (TWSTRS) and electrical activity from the trapezius and the sternocleidomastoid muscles measured by a surface electromyography (EMG).

Results and discussion: In the pre-post comparison patients showed significant improvements in the ROM (flexion/extension: 99.7 vs. 114.8 ; rotation: 105.5 vs. 123.6 ; lateral flexion: 56.6 vs. 70.9), physical health (SF-36 Score: 45.9 vs. 50.3) and TWSTRSScores (severity: 14.1 vs. 11.3; disability: 5.7 vs. 4.4 ; pain: 6.9 vs. 3.6). There was no significant improvement in patient's mental health and there were no significant changes in the EMG values.

In conclusion multimodal physiotherapy is a feasible and save additional treatment option for patients with cervical dystonia. An add-on physiotherapy program can achieve better treatment outcomes. 
Especially in range of motion, severity and pain patients benefited from the physiotherapy sessions.

\section{What do medical students know about deep brain stimulation?}

Wloch A. ${ }^{1}$, Lütjens G. ${ }^{1}$, Heissler H. ${ }^{1}$, Capelle H.H. ${ }^{1}$, Schrader C. ${ }^{2}$, Krauss J.K.

${ }^{1}$ Hannover Medical School, Department of Neurosurgery, Hannover, Germany, ${ }^{2}$ Hannover Medical School, Department of Neurology,

Hannover, Germany

Objective: DBS is a well-established therapy for movement disorders such as dystonia, Parkinson's disease (PD) and tremor and it is currently under investigation as a therapeutic option in neuropsychiatric disorders. Little is known about medical students' knowledge of this powerful tool when they enter university and what they learn about it during their medical formation. Thus, the objective here is to evaluate the knowledge of medical students about deep brain stimulation (DBS) at the beginning and at the end of their medical education.

Methods: A 10-item questionnaire with open and closed questions was designed. Questions addressed indications for DBS, its costs, impact on parkinsonian symptoms, complications, battery life, possible targets and percentage of PD patients who might profit from DBS. Students at Hannover Medical School were asked to complete the questionnaire in the preclinical study period and in the next to last year of the study.

Results: The group of "beginners" included 209 students (duration of study: 3 months) and the "advanced" group 170 students (duration of study: $48-72$ months). Of all students $63.6 \%$ were female and $36.4 \%$ were male. Age ranged between 17 and 46 years, mean 21.9 years in the first group and 25.1 years in the second. In the first group $22.7 \%$ had heard about DBS in medical lectures earlier and $15.7 \%$ had gained insight by television before. In comparison $98.76 \%$ had heard of DBS in medical lectures in the second group and knowledge was enhanced by television with $55.2 \%$. In group one $63.4 \%$ of the students knew that DBS is routinely used in PD patients as compared to $83.3 \%$ in the second group. Knowledge about other movement disorders was much less common. In the first group only $36.6 \%$ knew that DBS is a routinely used treatment for tremor, and only $10.3 \%$ knew that DBS is used in patients with dystonia, whereas $71.5 \%$ of students in the second group knew about the routine use of DBS in tremor but only one-third knew about its use in dystonia (34\%). Outcome after DBS, its costs, the frequency of side effects, and established and future targets were nearly unknown.

Conclusions: DBS is partly known among medical students in the preclinical phase with a moderate gain of knowledge during further study. We advocate to teach students more specifically and to expand clinical knowledge during the clinical phase of medical studies. Ref.Nr. 162-544685059-761.

\section{Complications of deep brain stimulation for secondary dystonia in the early postoperative period (30-day morbidity): an experience in 49 patients}

Wloch A. ${ }^{1}$, Abdallat M. ${ }^{1}$, Saryyeva A. ${ }^{1}$, Blahak C. ${ }^{2}$, Wolf J. ${ }^{3}$, Schrader C. ${ }^{4}$, Runge J. ${ }^{1}$, Krauss J.K. ${ }^{1}$
${ }^{1}$ Hannover Medical School, Department of Neurosurgery, Hannover, Germany, ${ }^{2}$ UMM, UniversitätsMedizin Mannheim, University of Heidelberg, Department of Neurology, Mannheim, Germany, ${ }^{3}$ Diakonissenkrankenhaus, Department of Neurology, Mannheim, Germany, ${ }^{4}$ Hannover Medical School, Department of Neurology, Hannover, Germany

Objective: Deep brain stimulation (DBS) has been shown to be efficacious in the treatment of primary dystonia. There is less experience in secondary dystonia. Since patients with secondary dystonia, who are often more disabled, may be more vulnerable to postoperative complications we aimed to investigate the 30-day morbidity in a large cohort of patients with secondary dystonia operated over a period of 19 years. Methods: From 1997 until 2016, a total of 49 patients [27 women and 22 men; mean age 43.5 years (range 13-77)] with secondary dystonia underwent DBS with electrodes implanted either in the Thalamus or the posteroventral lateral globus pallidus internus (GPi). Most frequent cause of for dystonia was cerebral palsy in 17 patients.

Results: There were no intraoperative or directly postoperative complications related to surgery. The electrode location was corrected in 2 instances. Two patients developed a wound infection, one patient had subdural hematoma and subcutaneous collection of cerebrospinal fluid (CSF) 3 weeks after DBS a subdural hematoma and subcutaneous collection of cerebrospinal fluid (CSF).

Conclusion: The 30-day morbidity rate in DBS for secondary dystonia is comparable to that in primary dystonia.

\section{Long term follow-up in Mohr-Tranebjaerg syndrome after pallidal stimulation}

Wloch A. ${ }^{1}$, Blahak C. ${ }^{2}$, Bäzner H. $^{3}$, Krauss J.K. ${ }^{1}$

${ }^{1}$ Hannover Medical School, Department of Neurosurgery, Hannover, Germany, ${ }^{2}$ UMM, UniversitätsMedizin Mannheim, University of Heidelberg, Department of Neurology, Mannheim, Germany, ${ }^{3}$ Klinikum Stuttgart, Department of Neurology, Stuttgart, Germany

Objective: Pallidal deep brain stimulation (DBS) has been established as a treatment option in patients with medically refractory dystonia. Mohr-Tranebjaerg syndrome (MTS) or Dystonia-Deafness-Syndrome is a rare genetic disorder characterized by deafness, dystonia and neurological abnormalities like impaired vision, dementia and cortical blindness. So far little is known about the efficacy of DBS in MTS.

Methods: A 44-year-old man with a history of generalized dystonia, deafness, visual blindness, ataxia and tremor was diagnosed with MTS which was confirmed by genetic analysis. He underwent bilateral stereotactic implantation of DBS electrodes in the posteroventral lateral globus pallidus internus (GPi). Electrode location was confirmed by postoperative stereotactic CT.

Results: Bilateral pallidal stimulation yielded modest improvement of dystonia at 6-months follow-up. During the next 4 months there was an increase of tremor and ataxia. The initial benefit was lost within the next 2 years. Extensive reprogramming did not yield additional improvement. After 3 years of chronic stimulation it was decided to switch off the pacemaker because of loss of efficacy.

\section{Hyperkinesias after long term pallidal stimulation for dystonia}

Wloch A. ${ }^{1}$, Lütjens G. ${ }^{1}$, Schrader C. ${ }^{2}$, Manu M. ${ }^{1}$, Blahak C. ${ }^{3}$ 
${ }^{1}$ Hannover Medical School, Department of Neurosurgery, Hannover, Germany, ${ }^{2}$ Hannover Medical School, Department of Neurology, Hannover, Germany, ${ }^{3}$ UMM, UniversitätsMedizin Mannheim, University of Heidelberg, Department of Neurology, Mannheim, Germany

Objective: The globus pallidus internus (GPi) is regarded as an established and safe target for deep brain stimulation (DBS) in dystonia. Recent reports on the occurrence of bradykinetic symptoms like freezing, postural instability and micrographia after chronic DBS of the GPi in patients with dystonia however stimulated a discussion on alternative targets. Here, we report on the unusual occurrence of dyskinesias upon chronic pallidal stimulation.

Methods: A 74-year-old man with segmental dystonia including blepharospasm, orofacial dystonia and aerophagia underwent bilateral stereotactic implantation of DBS electrodes in the GPi. Eleven years later during effective chronic DBS for dystonia he experienced gradual onset of hyperkinetic involuntary movements mainly concerning his arms but also his trunk.

Results: Detailed assessment in various conditions showed that off DBS resulted in an increase of both choreatic hyperkinesias and dystonia. High frequency and low amplitude DBS improved both dystonia and hyperkinesias, while high voltage DBS resulted in further improvement of dystonia but also in increased hyperkinesias. As a compromise between optimal stimulation for dystonia and hyperkinesias we finally choose a bipolar intermediate amplitude stimulation mode.

Conclusion: Chronic pallidal DBS might be accompanied not only by bradykinetic symptoms but also in the rare case by hyperkinesias. Such an occurrence requires complex reassessment of stimulation programming.

\section{Combined pallidal and thalamic stimulation as a therapy option for multifocal primary dystonia with prominent writers' cramp}

\author{
Wloch A. ${ }^{1}$, Abdallat M. ${ }^{1}$, Lütjens G. ${ }^{1}$, Schrader C. ${ }^{1}$, Krauss J.K. \\ ${ }^{1}$ Hannover Medical School, Departments of Neurosurgery \\ and Neurology, Hannover, Germany
}

Objective: The globus pallidus internus (GPi) has been established as the contemporary target of choice for deep brain stimulation (DBS) in dystonia. The thalamic Vim, however, has been preferred in patients with writer's cramp. When a patient presents with both writer's cramp and other dystonic symptoms, it is unclear which target should be chosen. Multifocal DBS is a new treatment option which can address this issue.

Methods: A 23-year- old woman with multifocal primary dystonia and prominent writers' cramp underwent bilateral stereotactic implantation of DBS electrodes in the GPi and the thalamic Vim. Electrode location was confirmed by postoperative stereotactic CT. All four electrodes were connected to an implantable pulse generator. Assessment included the Burke-Fahn- Marsden Dystonia Rating Scale (BFMDRS) motor and disability subscore, 36-item short-form health score (SF-36) and standard video recording. In the frame of a prospective study protocol clinical outcome was assessed before and at 3,15 and 24 months after surgery.

Results: Thalamic stimulation yielded an improvement in writer's cramp of about $80 \%$ according to the BFMDRS subscores. At 3 months follow-up the BFMDRS motor subscore had decreased from 3 before surgery to 0 and the disability subscore had decreased from 6 to 2. At the same time the SF-36 had improved from 31 to 78 . The effect lasted for 1 year. At 15 months follow-up the BFM motor subscore had increased to 10 and the disability subscore had increased to 6 . The SF-36 had decreased to 67 . The patient presented with an increase in dystonia of the right hand and the right foot accompanied by pain. Using bilateral and monopolar Vim thalamic stimulation did not have a marked impact in the severity of dystonia. Bilateral GPi stimulation alone improved slightly the dystonic symptoms in the right foot but did not address the writer's cramp. Because the patient suffered in particularly right-sided dystonia a combined contralateral thalamic and pallidal stimulation was chosen. We saw an improvement almost immediately of both the writer's cramp and the dystonic foot after the initiation of the combined unilateral bipolar stimulation. The BFM motor subscore had decreased to 6 , the disability subscore had decreased to 4 and SF-36 remained unchanged with 65 . The effect was sustained at 24 month follow-up. The combination of pallidal and thalamic stimulation provided finally an improvement of $40 \%$ in BFM motor subscore.

Conclusion: Combined thalamic and pallidal stimulation in patients with multifocal primary dystonia under certain circumstances might be an emergent therapy option.

\section{Stable symptom improvement after battery depletion in a patient with deep brain stimulation for secondary dystonia}

Wolf, Marc $^{1}$; Blahak, Christian ${ }^{1}$; Schrader, Christoph ${ }^{2}$; Krauss, Joachim $^{3}$

${ }^{1}$ Universitaets Medizin Mannheim, University of Heidelberg, Department of Neurology, Mannheim, Germany, ${ }^{2}$ Medical School Hannover, Department of Neurology, Hannover, Germany, ${ }^{3}$ Medical School Hannover, Department of Neurosurgery, Hannover, Germany

Background: In long-term studies, sustained clinical benefit of DBS in dystonia has been reported. To maintain this effect, patients need regularly replacements of the implantable pulse generator (IPG) generally performed before complete battery depletion to avoid temporary deterioration of dystonic symptoms.

Case report: At the age of 25 , a male patient with generalized secondary dystonia and choreatiform movements due to perinatal asphyxia underwent bilateral DBS of the ventral intermediate nucleus (Vim) of the thalamus and the posteroventral lateral globus pallidus internus (GPi). Initially IPG was connected to Vim-DBS electrodes. Preoperative clinical examination showed an actioninduced myocloniform tremor and dystonia with additional spastic tetraparesis and ataxic gait, requiring a wheelchair for longer distances. The patient was not able to drink from a glass by himself and needed assistance for eating (BFM 80.5). Post-operatively, dystonic tremor of the upper extremities clearly improved (BFM 67.5). Fourty months postoperatively the patient reported slowly progressing dystonic symptoms, while the IPG revealed to be switched-off since several months. Stimulation was changed to GPi at IPG replacement leading to a noticeable improvement of gait with continuing good control of dystonic tremor. At 76 months after DBS implantation, the patient presented for his routine follow-up with stable clinical symptoms, whereas DBS again revealed to be switched off since 5 weeks.

Conclusions: In 2 studies that systematically analysed the re-occurrence of dystonic symptoms 24/48 h following DBS cessation, dystonia did not reach the preoperative severity but remained at an intermediate level in the majority of patients. All had DBS for several years. Our patient had an extremely sustained clinical benefit exceeding earlier expectations following DBS discontinuation. He had DBS for many years and was sequentially stimulated at two different sites, both of these aspects might play a role for 
persisting modulatory effect on the motor network following DBS cessation.

\section{Problems of the quality of life in patients with tardive dyskinesia}

\author{
Zakharov D. ${ }^{1}$, Khublarova L. ${ }^{1}$ \\ ${ }^{1}$ Federal State Budgetary Institution "Bekhterev Psychoneurological \\ Research Institute in St. Petersburg”, Saint-Petersburg, Russian \\ Federation
}

Involuntary movements that develop on the background of drug therapy can cause serious psychosocial consequences in patients. Thus, these patients find it difficult not only because of their disease, but also because of the poor orientation of the society to the disease. The latter causes a constant feeling of shame, guilt, anxiety, and anger, and leads to reduced quality of life.

The objective of this study was to evaluate the quality of life in patients with neuroleptic complications.

Materials and methods: The study conducted on the basis of our site involved 40 subjects ( 28 women, 12 men, aged from 23 to 76 years) with verified diagnosis of "schizophrenia" in the state of remission according to the ICD-10, with signs of late complications and receiving neuroleptic antipsychotics for 12-216 months. Control group involved 30 subjects with verified diagnosis of "schizophrenia" without tardive dyskinesia. The quality of life was assessed using the SF-36 questionnaire.

Results: We have convincingly shown that these patients with dyskinesia have a significant reduction in the quality of life compared with the control group.

Conclusions: All of the above points complicate the course of the underlying disease, increasing the severity of the negative and affective disorders. These involuntary movements and mental illness cause a marked reduction of quality of life.

This points to the obvious relevance of studying the problem and the need to find new successful methods for correction of neuroleptic complications.

\section{Botulinum therapy offered for the patients with tardive form of neuroleptic dyskinesia}

\author{
Zakharov D. ${ }^{1}$, Khublarova L. ${ }^{1}$, Mikhaylov V. ${ }^{1}$ \\ ${ }^{1}$ St. Petersburg Bekhterev Psychoneurological Research Institute, \\ Saint-Petersburg, Russian Federation
}

Tardive neuroleptic dystonia is one of the most severe forms of medical dyskinesias with poor response to the treatment. The severity of neuroleptic side effects for patient often leads to a poor compliance, which, in turn, often provokes an exacerbation of the endogenous process.

The aim of this work is to study the effectiveness of botulinum therapy with patients with tardive form of neuroleptic dyskinesia.

Materials and methods: 40 patients were included in the study group with tardive neuroleptic complications, receiving neuroleptic antipsychotics during 18-216 months. All the patients had tardive neuroleptic complications appeared with dyskinesias predominantly in the facial or neck muscles. All the patients received botulinum toxin type A (Xeomin) which dose was individually selected from 70 to $300 \mathrm{ED}$.

Results: After the botulinum toxin treatment, all the patients noticed positive dynamics of their condition. According to the Clinical Global
Impression rating scale treatment results were predominantly rated as good and very good. Moreover, the results of Simpson scale showed a decrease from 78.6 to 59.6; according to Simpson Angus rating scale for extrapyramidal side effects there was a decrease with the total score from 7.3 to 5.2. The same results we observed through Abnormal Involuntary Movement Scale (AIMS) that suggested a decrease with total score from 21.1 to 13.5. The patients with cervical dystonia demonstrated maximum improvement. Minimum clinical improvement was observed with elderly patients, the people suffering from dyskinesia with incomplete dentition, or the lack of it. It should be mentioned that unsatisfactory effect observed among the patients with severe hyperkinesis of the tongue, which is obviously caused by technical complexity of the injection as well.

Conclusions: All in all, the botulinum therapy seems to be one of the most effective methods of the present time, to treat tardive neuroleptic dyskinesias.

\section{Dopamine, reversal learning and decision making: Enhanced cognitive control in task-specific dystonia}

\author{
Zeuner K. ${ }^{1}$, Knutzen A. ${ }^{1}$, Granert O. ${ }^{1}$, Sablowsky S. ${ }^{1}$, Dressler D. ${ }^{2}$, \\ Klein $\mathrm{C}^{3}$, Witt K. \\ ${ }^{1}$ University of Kiel, Department of Neurology, Kiel, Germany, \\ ${ }^{2}$ Movement Disorders Section, Department of Neurology, Hannover \\ Medical School, Hannover, Germany, ${ }^{3}$ Institute of Neurogenetics, \\ University of Lübeck, Lübeck, Germany
}

Background: In task specific dystonia previous receptor binding studies suggest that dopamine signaling within the basal ganglia circuits is abnormal. Furthermore, the DRD2/ANKK1-TaqIa polymorphism is associated with $30 \%$ reduction of the striatal dopamine receptor density with reward-based learning and is impaired in A + subjects.

Method: Reward-based learning was compared between 31 writer's cramp patients and 35 controls using fMRI. Subjects chose in a probabilistic reversal-learning task between two stimuli and indicated their response with their left/right index finger. At first, one stimulus response was rewarded in $80 \%$ (smiling face). A sad face was presented in $20 \%$ for the second stimulus. After 14-18 blocks the contingencies reversed, and the second stimulus response was rewarded in $80 \%$. We further linked the DRD2/ANKK1-TaqIa polymorphism.

Results: Feedback learning in patients was normal. Patients showed greater increase in blood-oxygen level dependent (BOLD) signal activity following negative feedback in the middle anterior cingulate cortex (BA 32). The genetic status was not correlated with the BOLD activity.

Conclusion: Brodmann area 32 (BA32) has an important role in reward-based learning and is connected with the basal ganglia-thalamo-loop modulated by dopaminergic signalling. This finding suggests disturbed integration of reinforcement history when dopamine is implicated in decision making. The results provide evidence for a paradigm implicating dysfunction in the prefrontal cortex in task specific dystonia.

Open Access This article is distributed under the terms of the Creative Commons Attribution 4.0 International License ( http://creativecommons.org/licenses/by/4.0/), which permits unrestricted use, distribution, and reproduction in any medium, provided you give appropriate credit to the original author(s) and the source, provide a link to the Creative Commons license, and indicate if changes were made. 